\title{
Analytical solutions for the surface response to small amplitude perturbations in boundary data in the shallow-ice-stream approximation
}

\author{
G. H. Gudmundsson \\ British Antarctic Survey, High Cross, Madingley Rd., Cambridge CB3 OET, UK \\ Received: 19 November 2007 - Published in The Cryosphere Discuss.: 11 January 2008 \\ Revised: 30 May 2008 - Accepted: 13 June 2008 - Published: 16 July 2008
}

\begin{abstract}
New analytical solutions describing the effects of small-amplitude perturbations in boundary data on flow in the shallow-ice-stream approximation are presented. These solutions are valid for a non-linear Weertman-type sliding law and for Newtonian ice rheology. Comparison is made with corresponding solutions of the shallow-ice-sheet approximation, and with solutions of the full Stokes equations. The shallow-ice-stream approximation is commonly used to describe large-scale ice stream flow over a weak bed, while the shallow-ice-sheet approximation forms the basis of most current large-scale ice sheet models. It is found that the shallow-ice-stream approximation overestimates the effects of bed topography perturbations on surface profile for wavelengths less than about 5 to 10 ice thicknesses, the exact number depending on values of surface slope and slip ratio. For high slip ratios, the shallow-ice-stream approximation gives a very simple description of the relationship between bed and surface topography, with the corresponding transfer amplitudes being close to unity for any given wavelength. The shallow-ice-stream estimates for the timescales that govern the transient response of ice streams to external perturbations are considerably more accurate than those based on the shallow-ice-sheet approximation. In particular, in contrast to the shallow-ice-sheet approximation, the shallow-ice-stream approximation correctly reproduces the short-wavelength limit of the kinematic phase speed given by solving a linearised version of the full Stokes system. In accordance with the full Stokes solutions, the shallow-icesheet approximation predicts surface fields to react weakly to spatial variations in basal slipperiness with wavelengths less than about 10 to 20 ice thicknesses.
\end{abstract}

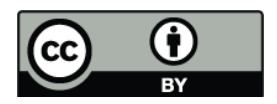

Correspondence to:

G. H. Gudmundsson (ghg@ @as.ac.uk)

\section{Introduction}

Large-scale ice sheet models commonly employ approximations to the momentum equations for increased computational efficiency. These approximations are derived from the full-set of momentum equations through scaling analysis motivated by the size of some geometrical aspect ratios, such as ice thickness and ice-sheet span, and some expectations about relative sizes of various stress terms. Currently, most large-scale ice sheet models are based on two different types of approximations, which in this paper will be referred to as the shallow-ice-sheet (SSHEET) and the shallow-icestream (SSTREAM) approximations. The shallow-ice-sheet approximation corresponds to the situation where surfaceparallel shear stress in a shallow ice sheet are large compared to horizontal deviatoric stress. The shallow-ice-stream approximation, on the other hand, assumes that the vertical shear stress is small compared to all other stress components. Both of these approximations use the shallow-ice approximation, i.e. they describe flow over horizontal scales large compared to the mean ice thickness. Derivations of the resulting theories, often using slightly different scaling arguments, can be found, for example, in Hutter (1983), Morland (1984), Muszynski and Birchfield (1987), MacAyeal (1989), and Baral and Hutter (2001).

It is clearly of considerable importance to be able to quantify the errors introduced by these different approximations. The derivations of the SSHEET and the SSTREAM approximations give some indications about their applicability to different flow regimes. It follows, for example, from the scalings used in the SSTREAM approximation that the slip ratio, the ratio between mean basal motion and mean forward deformational velocity, must be $O\left(\delta^{-2}\right)$, where $\delta$ is the ratio between typical thickness and horizontal span (see Appendix A). The errors are also expected to be of some order of the

Published by Copernicus Publications on behalf of the European Geosciences Union. 
ratio between typical ice-thickness and horizontal scales of the problem. For example, for the SSTREAM approximation the errors are $O\left(\delta^{2}\right)$. For a given problem of interest to a modeller it is, however, generally difficult if not impossible to come up with firm quantitative estimates of those errors.

A straightforward possibility of assessing the applicability of these approximations to situations commonly encountered in glaciology is to compare solutions to those obtained by using the full-system momentum-balance equations (FS solutions). However, although possible in principle, the computational cost of a FS solution makes this approach, in most cases, impractical. A promising solution to this problem has been suggested by Hindmarsh (2004) who performed a computational analysis of various approximations used in glaciology by comparing flow disturbances set up by small perturbations in the ice surface. Focusing on small-amplitude perturbations reduces computational times making direct estimates of absolute errors feasible. Another advantage that comes from analysing small-amplitude solutions is the added insight they can give into the nature of the approximations. From the scaling analysis of the SSTREAM approximation it is, for example, far from obvious how the relationship between bed and surface differs from that given by the SSHEET approximation, and how those descriptions in turn differ from the one given by the FS theory. One of the key advantages to come from analysing effects of small-amplitude perturbations on flow is that by doing so fairly general answers to these questions can be given.

Here I present new analytical solutions to the shallow SSTREAM equations based on small-amplitude perturbation analysis and compare them with corresponding FS analytical solutions given in Gudmundsson (2003) and Jóhannesson (1992). Comparisons with analytical solutions based on the SSHEET approximation are also made. The solutions are valid for linear medium and small-amplitude perturbations in surface topography, bed topography, and basal slipperiness.

\section{Linear perturbation analysis of the shallow-ice- stream approximation}

The method of comparing these solutions followed here is to cast them in the form of transfer functions. These transfer functions describe the transient response of the medium to perturbations in bed topography, surface geometry, and basal slipperiness. The starting point is the well-known shallow-ice-stream equations (MacAyeal, 1989). The derivation of these equations is outlined in Appendix A. Further examples of their derivations, using slightly different scaling arguments, can be found elsewhere (e.g. MacAyeal, 1989; Schoof, 2006).

The analysis is done in a coordinate system tilted forward in the $x$ direction by the angle $\alpha$, the mean surface slope. As shown in Appendix A the shallow-ice-stream scalings results in

$$
\begin{aligned}
& \partial_{x}\left(4 h \eta \partial_{x} u+2 h \eta \partial_{y} v\right)+\partial_{y}\left(h \eta\left(\partial_{x} v+\partial_{y} u\right)\right)-(u / c)^{1 / m} \\
& \quad=\rho g h \partial_{x} s \cos \alpha-\rho g h \sin \alpha, \\
& \partial_{y}\left(4 h \eta \partial_{y} v+2 h \eta \partial_{x} u\right)+\partial_{x}\left(h \eta\left(\partial_{y} u+\partial_{x} v\right)\right)-(v / c)^{1 / m} \\
& \quad=\rho g h \partial_{y} s \cos \alpha,
\end{aligned}
$$

which are two coupled partial differential equations for the depth-independent horizontal velocity components $u$ and $v$. In these equation $s$ is the surface, $h$ is the ice thickness, $\eta$ is the effective ice viscosity, $\rho$ the ice density, and $c$ is the basal slipperiness. The parameter $m$ and the basal slipperiness $c$ are parameters in the sliding law defined by Eq. (A5) in Appendix A.

For a linear viscous medium $(n=1)$ and a non-linear sliding law ( $m$ arbitrary but positive) these equations can be linearised and solved analytically using standard methods. We write $f=\bar{f}+\Delta f$, where $f$ stands for some relevant variable entering the problem, and look for a zeroth-order solution where $\bar{f}$ is independent of $x$ and $y$ and time $t$, while the firstorder field $\Delta f$ is small but can be a function of space and time.

The perturbations in bed topography $(\Delta b)$ and basal slipperiness $(\Delta c)$ are step functions of time. They are applied at $t=0$, i.e. for $t<0$ we have $\Delta b=0$ and $\Delta c=0$. For $t \geq 0$ both $\Delta b$ and $\Delta c$ are some nonzero functions of $x$ and $y$. Using this history definition, the solutions for the velocity field and the surface geometry become functions of time. The purpose of introducing time varying basal perturbations is to be able to study the transient response of the surface to temporal changes in basal conditions. As shown below the transient response is determined by two timescales: the phase time scale $\left(t_{p}\right)$, and the relaxation time scale $\left(t_{r}\right)$. Both of these timescales are independent of the particular time history used for the basal perturbations. They are also independent of the nature of the basal perturbation, i.e. exactly the same expressions are obtained for $t_{p}$ and $t_{r}$ for a perturbation in basal topography as for a perturbation in basal slipperiness. Using the superposition principle, solutions for more complicated time histories can easily be constructed.

\subsection{Bed topography perturbations}

We start by considering the response to small perturbation in basal topography $(\Delta b)$. Writing $h=\bar{h}+\Delta h, s=\bar{s}+\Delta s$, $b=\bar{b}+\Delta b$, where $h$ is ice thickness, $s$ surface topography, and $b$ bed topography, and $u=\bar{u}+\Delta u, v=\Delta v, w=\Delta w$, where $u, v$, and $w$ are the $x, y$, and $z$ components of the velocity vector, respectively, and $c=\bar{c}$ where $c$ is the basal slipperiness (see Eq. A5), inserting into (1) and (2) and solving the resulting equations gives the zeroth-order solution

$\bar{u}=\bar{c} \rho g \bar{h} \sin \alpha$. 
The zeroth-order solution represents a plug flow down an uniformly inclined plane.

The first-order field equations are

$$
\begin{aligned}
& 4 \eta \bar{h} \partial_{x x}^{2} \Delta u+3 \eta \bar{h} \partial_{x y}^{2} \Delta V+\eta \bar{h} \partial_{y y}^{2} \Delta u-\gamma \Delta u \\
& =\rho g \bar{h} \cos \alpha \partial_{x} \Delta s-\rho g \sin \alpha \Delta h,
\end{aligned}
$$

and

$$
\begin{aligned}
& 4 \eta \bar{h} \partial_{y y}^{2} \Delta v+3 \eta \bar{h} \partial_{x y}^{2} \Delta u+\eta \bar{h} \partial_{x x}^{2} \Delta v-\gamma \Delta v \\
& \quad=\rho g \bar{h} \cos \alpha \partial_{y} \Delta s,
\end{aligned}
$$

where

$$
\gamma=\frac{\tau_{d}^{1-m}}{m \bar{c}}
$$

and

$\tau_{d}=\rho g \bar{h} \sin \alpha$,

is the driving stress.

The domain of the first-order solution is transformed to that of the zeroth-order problem. This is done by writing $f=\bar{f}+\Delta f+\partial_{z} \bar{f} \Delta z$ where $f$ is any given term that enters the boundary conditions, and $\Delta z$ is either $\Delta s$ or $\Delta b$.

To first order, the upper and lower boundary kinematic conditions are

$\partial_{t} \Delta s+\bar{u} \partial_{x} \Delta s-\Delta w=0$

and

$\bar{u} \partial_{x} \Delta b-\Delta w=0$,

respectively. In (8) the surface mass-balance perturbation has been set to zero. The jump conditions for the stress tensor have already been used in the derivation of (1) and (2) and do not need to be considered further.

This system of equations is solved using standard Fourier and Laplace transform methods. All variables are Fourier transformed with respect to the spatial variables $x$ and $y$ and Laplace transformed with respect to the time variable $t$. In the forward Fourier transform the two space variables both carry a positive sign, and the wavenumbers in $x$ and $y$ direction are denoted by $k$ and $l$, respectively. In the forward Laplace transform the time variable carries a negative sign, and the complex Laplace argument is denoted by the variable $r$.

The Fourier and Laplace transforms of the first order field Eqs. (4) and (5) are

$$
\begin{gathered}
4 \eta \bar{h} k^{2} \Delta u+3 \eta \bar{h} k l \Delta v+\eta \bar{h} l^{2} \Delta u+\gamma \Delta u \\
=\rho g \sin \alpha(\Delta s-\Delta b)+i k \rho g \bar{h} \Delta s \cos \alpha,
\end{gathered}
$$

and

$$
\begin{aligned}
& 4 \eta \bar{h} l^{2} \Delta v+3 \eta \bar{h} k l \Delta u+\eta \bar{h} k^{2} \Delta v+\gamma \Delta v \\
& =\mathrm{i} l \rho g \bar{h} \Delta s \cos \alpha,
\end{aligned}
$$

respectively. The Fourier transformed mass-conservation equation is

$-\mathrm{i} k \Delta u-\mathrm{i} l \Delta v+\partial_{z} \Delta w=0$.

Equations (10) to (12) can now be solved for $\Delta u, \Delta v$ and $\partial_{z} \Delta w$. Vertical integration of $\partial_{z} \Delta w$ and insertion into the kinematic boundary condition at the surface

$\Delta w(\bar{s})=(r-\mathrm{i} k \bar{u}) \Delta s$,

gives the surface response, and after some simple algebraic manipulations, one finds that the (complex) ratio between surface and bed amplitudes $T_{s b}=\Delta s / \Delta b$ is given by

$T_{s b}(k, l, r)=-\frac{\mathrm{i} k\left(\bar{u}+\tau_{d} / \xi\right)}{r(r-p)}$,

where

$p=\mathrm{i} / t_{p}-1 / t_{r}$,

and the two timescales $t_{p}$ and $t_{r}$ are given by

$t_{p}^{-1}=k\left(\bar{u}+\tau_{d} / \xi\right)$,

and

$t_{r}^{-1}=\xi^{-1} j^{2} \tau_{d} \bar{h} \cot \alpha$,

and where furthermore the two abbreviations

$\xi=\gamma+4 \bar{h} j^{2} \eta$

and

$j^{2}=k^{2}+l^{2}$,

have been used.

An inverse Laplace transform of Eq. (14) using contour integration leads to

$T_{s b}(k, l, t)=\frac{\mathrm{i} k\left(\bar{u} \xi+\tau_{d}\right)}{p \xi}\left(e^{p t}-1\right)$.

This transfer function describes the relation between surface and bed topography, where

$\Delta s(k, l, t)=T_{s b}(k, l, t) \Delta b(k, l)$.

Other transfer functions are defined in an analogous manner.

In Gudmundsson (2003) the relaxation time scale is referred to as the decay time scale and denoted by $t_{d}$. As pointed out by Hindmarsh (2004), depending on the situation, the term "growth rate" is presumably more descriptive. Here the term "relaxation time scale" will be used as the time scale $t_{r}$ determines how long it takes for the transient solution to "relax" toward the steady-state limit.

The relationship between surface velocity and bed topography is found to be given by

$T_{w b}=k\left(k \bar{u}-e^{p t}(\mathrm{i} p+k \bar{u})\right)\left(\bar{u} \xi+\tau_{d}\right)(p \xi)^{-1}$, 
for the vertical velocity component $(w)$, and

$$
\begin{gathered}
T_{u b}=-\frac{p\left(\gamma+\bar{h}\left(k^{2}+4 l^{2}\right)\right) e^{p t}}{\bar{h} p \nu \xi} \\
+\frac{\tau_{d} \bar{h} \cot \alpha\left(l^{2} \tau_{d}-k^{2} \bar{u} \nu\right)\left(1-e^{p t}\right)}{\bar{h} p \nu \xi},
\end{gathered}
$$

and

$T_{v b}=\frac{k l \tau_{d}\left(3 p \eta e^{p t}+\left(e^{p t}-1\right)\left(\bar{u} \nu+\tau_{d}\right) \cot \alpha\right)}{p \nu \xi}$,

where

$v=\gamma+\bar{h} j^{2} \eta$,

for the longitudinal $(u)$ and the transverse $(v)$ components, respectively.

\subsection{Perturbations in basal slipperiness}

Transfer functions describing the effects of spatial variations in basal slipperiness on surface geometry and surface velocities can be derived in a similar fashion. In Appendix B the solution procedure is outlined and expressions for the corresponding transfer functions listed.

\subsection{Surface perturbations}

We now determine the transient evolution of a surface undulation prescribed at $t=0$. By writing $h=\bar{h}+\Delta s, s=\bar{s}+\Delta s$, $b=\bar{b}, u=\bar{u}+\Delta u, v=\Delta v, w=\Delta w$, and $c=\bar{c}$, inserting into (1) and (2) and solving the resulting equations together with (12) and the kinematic boundary condition at the surface

$w=r \Delta s-\mathrm{i} k \bar{u} \Delta s-s(t=0)$,

it is found that the surface evolution as a function of time is given by

$s(k, l, t)=T_{s s_{0}} s(k, l, t=0)$,

where

$T_{s s_{0}}=e^{p t}$.

The velocity components are given by

$T_{w s_{0}}=\left(\mathrm{i} k-j^{2} \bar{h} \cot \alpha\right) \tau_{d} \xi^{-1} e^{p t}$,

$T_{u s_{0}}=\frac{\tau_{d}\left(\gamma(1+\psi)+\bar{h} \eta\left(j^{2} \psi+k^{2}+4 l^{2}\right)\right)}{\bar{h} \nu \xi} e^{p t}$,

where

$\psi=\mathrm{i} k \bar{h} \cot \alpha$,

and

$T_{v s_{0}}=\frac{\mathrm{i} l(3 \mathrm{i} k \eta+v \cot \alpha) \tau_{d}}{\nu \xi} e^{p t}$.

\subsection{Non-dimensional forms of the transfer functions}

The transfer functions listed above are all in dimensional form. It is often much more convenient to work with the transfer functions in a non-dimensional form. For this purpose the same scalings as used in Gudmundsson (2003) will be employed. The velocity is given in units of mean deformational velocity of the full-system solution. All spatial scales are in units of mean ice thickness $(\bar{h})$, and stress components in units of driving stress $\left(\tau_{d}\right)$. It follows from these scalings that the non-dimensional viscosity, $E$, is given by $E=\bar{u}_{d} \eta /\left(\bar{h} \tau_{d}\right)=1 / 2$, and the mean non-dimensional basal slipperiness $\bar{C}$ is

$\bar{C}=\bar{c} \tau_{d}^{m} / \bar{u}_{d}=\bar{u}_{b} / \bar{u}_{d}$.

The mean non-dimensional slipperiness is therefore equal to the slip ratio, i.e. the ratio between mean basal sliding velocity $\left(\bar{u}_{b}\right)$ and the mean forward deformational velocity $\left(\bar{u}_{d}\right)$. One obtains the non-dimensional form of the transfer functions from the dimensional one using the substitutions $\bar{c} \mapsto \bar{C}, \eta \mapsto 1 / 2, \bar{h} \mapsto 1, \bar{u} \mapsto \bar{C}, k \mapsto k, l \mapsto l$, $\gamma \mapsto(m \bar{C})^{-1}$, and $\rho g \bar{h} \sin \alpha \mapsto 1$.

Note that since in the shallow-ice-stream approximation $\bar{u}_{d}=O\left(\delta^{2}\right)$ and we are ignoring all fields to this order, we have $\bar{u} \mapsto C$ and not $\bar{u} \mapsto C+1$ as is the case for the fullsystem solutions and the solutions of the shallow-ice-sheet approximation. Furthermore, from $\bar{C}=\bar{u}_{b} / \bar{u}_{d}$ it follows that $\bar{C}=O\left(\delta^{-2}\right)$. In these non-dimensional units, the requirement that the slip ratio is $O\left(\delta^{-2}\right)$ for the shallow-icestream approximation to be valid implies $\bar{C}+1 \approx \bar{C}$ or $\bar{C} \gg 1$ for small surface slopes.

In non-dimensional form the solutions often take a considerably simpler shape. For example the non-dimensional forms of the timescales $t_{p}$ and $t_{r}$, and the transfer function $T_{s b}$ are, respectively,

$t_{r}=\left(2+\left(j^{2} m \bar{C}\right)^{-1}\right) \tan \alpha$,

$t_{p}^{-1}=k \bar{C}\left(1+\frac{m}{1+2 j^{2} m \bar{C}}\right)$,

and

$T_{s b}=\frac{k\left(1+\left(1+2 j^{2} \bar{C}\right) m\right)}{k+m\left(k+2 k j^{2} \bar{C}+\mathrm{i} j^{2} \cot \alpha\right)}\left(1-e^{\mathrm{i} t / t_{p}} e^{-t / t_{r}}\right)$.

Note that in all of these three expressions, the short wavelength limit $(k$ and $l \rightarrow+\infty)$ is independent of the sliding law exponent $m$.

\section{Discussion}

The main subject of the following discussion is a comparison of various SSHEET, SSTREAM and FS small-amplitude solutions. The SSHEET solutions are known for non-linear medium and a non-linear sliding law (Nye, 1960; Fowler, 


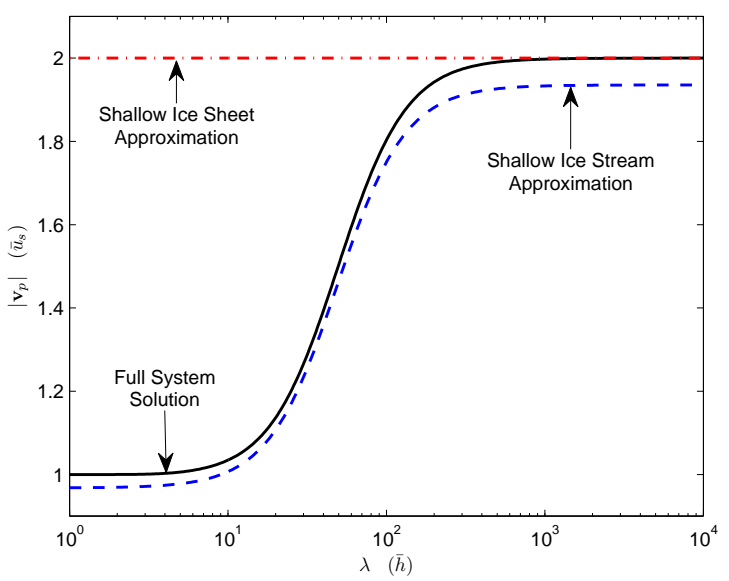

Fig. 1a. The phase speed $\left(\left|\mathbf{v}_{p}\right|\right)$ as a function of wavelength for $\theta=0$. The dashed-dotted curve is based on the shallow-ice-sheet (SSHEET) approximation, the dashed one is based on the shallowice-stream (SSTREAM) approximation, and the solid one is a fullsystem (FS) solution. The surface slope is $\alpha=0.005$ and slip ratio $\bar{C}=30$ and $n=m=1$. The unit on the y axis is the mean surface velocity of the full-system solution $(\bar{u}=\bar{C}+1=31)$.

1982; Jóhannesson, 1992). Small-amplitude FS solutions have so far only been derived for linear medium and a linear sliding law (Reeh, 1987; Jóhannesson, 1992; Gudmundsson, 2003). The SSTREAM solutions, derived here for the first time, are valid for linear medium and a non-linear sliding law. It follows that direct comparision between the SSTREAM and the FS solutions can only be done for $n=1$ and $m=1$, and for this reason most of the discussion is limited to this case. The only exception is a brief description given below of the relationship between bed and surface geometry in the SSTREAM approximation for non-linear sliding law. A full discussion of the non-linear aspects of the solutions will be done elsewhere.

\subsection{Time scales}

As seen from the solutions listed above and in Appendix B the transient behaviour is completely determined by two time scales: the phase time scale $t_{p}$, and the relaxation time scale $t_{r}$. The term "phase time scale" is used for $t_{p}$ because it determines how quickly the phase of the surface fields changes with time (see for example Eqs. 26 and 27). As mentioned above the $t_{r}$ time scale determines how quickly the solutions "relax" toward the steady-state limit.

The properties of the phase time scale can most easily be understood by looking at the phase velocity $\mathbf{v}_{p}$ which is, by definition, given by $\mathbf{v}_{p}=\left(\left(k t_{p}\right)^{-1},\left(l t_{p}\right)^{-1}\right)$. The SSTREAM phase speed is

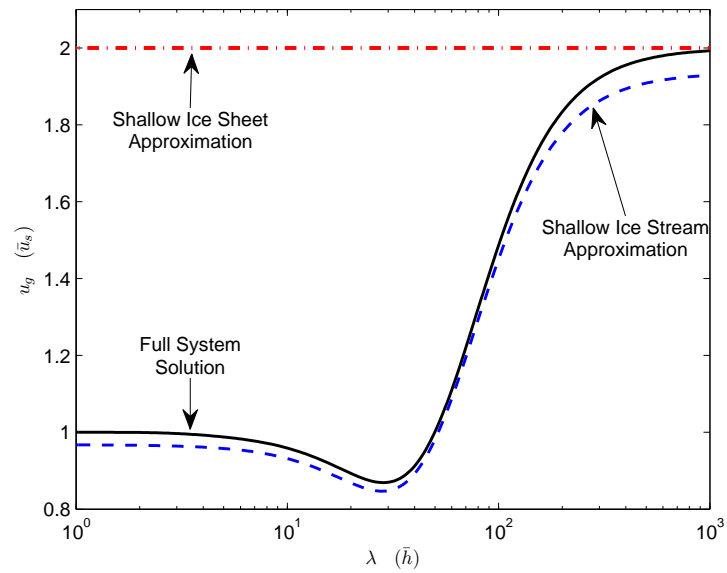

Fig. 1b. The $x$ component of the group velocity $\left(u_{g}\right)$ as a function of wavelength for $\theta=0$. Values of mean surface slope and slip ratio are 0.005 and 30 , respectively, and $m=n=1$.

$\left|\mathbf{v}_{p}\right|=\left(j t_{p}\right)^{-1}=\cos \theta\left(\bar{u}+\frac{\tau_{d}}{\gamma+4 j^{2} \bar{h} \eta}\right)$,

in dimensional units. The angle $\theta$ is the angle between the wave vector $\mathbf{k}=(k, l)$ and the $\mathrm{x}$ axis. This is also the angle between the $x$ axis and a vector lying in the $x y$ plane and normal to the crests of the sinusoidal perturbations. We have $\cos \theta=k / j$ and $\lambda=2 \pi / j$ where $\lambda$ is the wavelength.

It is instructive to compare phase speeds for different approximations. Figure 1a shows the phase speed for $\theta=0$ as a function of wavelength for the shallow-ice-stream approximation (dashed line), the shallow-ice-sheet approximation (dashed-dotted line), and for the full-system solution (solid line) for $m=1$ and $n=1$. Note that in the figure the phase speeds have been normalised by the surface velocity as given by the full-system solution (equal to $\bar{C}+1$ in non dimensional units).

In the shallow-ice-sheet (SSHEET) approximation the phase speed (dashed-dotted curve in Fig. 1a) is, for $\theta$ fixed, independent of the wavelength. For $\theta=0$ the SSHEET phase speed is $(n+1) u_{d}+(m+1) u_{b}$, where $u_{d}$ is the deformational velocity and $u_{b}$ the basal sliding velocity. This is a well known (Nye, 1960) and often used expression for the speed of surface waves of on glaciers. The long-wavelength limit of the SSHEET phase speed is correct, but the short wavelength SSHEET limit is incorrect. The FS limit for $\theta=0$ as $\lambda \rightarrow 0$ is $\left|\mathbf{v}_{p}\right|=u_{d}+u_{b}$, i.e. for short wavelengths the kinematic wave speed equals the surface velocity.

As can be seen from direct inspection of Eqs. (32) and Eq. (90) in Gudmundsson (2003) - giving $\left|\mathbf{v}_{p}\right|$ for the SSTREAM and the FS theories, respectively - and as illustrated by Fig. 1a, the SSTREAM phase speed (dashed curve) is quite similar to the FS phase speed for $\bar{C} \gg 1$. In particular, in contrast to the SSHEET phase speed, the SSTREAM 


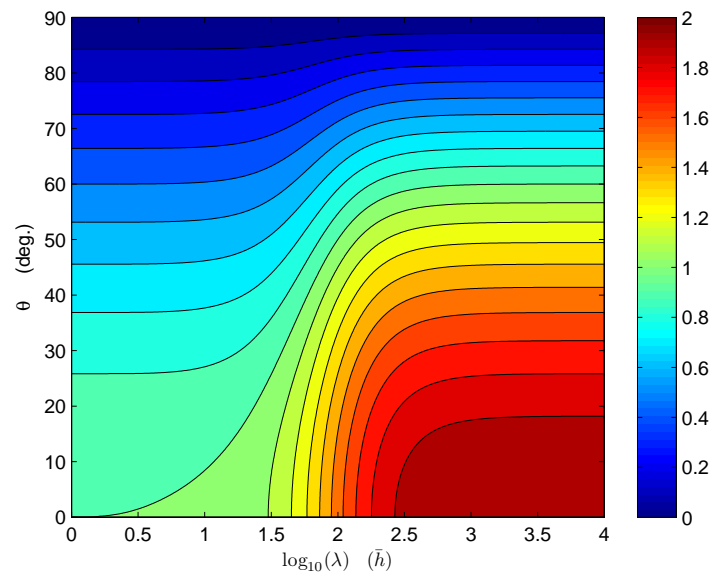

Fig. 2a. The phase speed $\left(\left|\mathbf{v}_{p}\right|\right)$ of the full-system solution as a function of wavelength $\lambda$ and orientation $\theta$ of the sinusoidal perturbations with respect to mean flow direction. The mean surface slope is $\alpha=0.002$ and the slip ratio is $\bar{C}=100$, and $n=m=1$. The plot has been normalised with the non-dimensional surface velocity $\bar{u}=\bar{C}+1=101$ of the full-system solution.

phase speed given by Eq. (32), valid for any positive $m$ and $n=1$, is not independent of $\lambda$. The $\lambda \rightarrow+\infty$ SSTREAM limit is $\left|\mathbf{v}_{p}\right|=\bar{u}(1+m) \cos \theta$, which agrees with the FS limit for $u_{d}=0$. For $\lambda \rightarrow 0$ the SSTREAM phase speed is equal to the mean surface speed, again in an agreement with the FS theory. Hence, in both the $\lambda \rightarrow 0$ and the $\lambda \rightarrow+\infty$ limits, the SSHEET phase speed is equal to the FS phase speed.

The differences between the FS and the SSTREAM phase speeds are small for parameter values typical of an active ice stream. This can be seen most easily from direct inspection of the differences between $t_{p}$ given by Eq. (16) and Eq. (73) in Gudmundsson (2003). Figures $2 \mathrm{a}$ and $\mathrm{b}$ show the FS and the SSTREAM phase speeds $\left(\left|\mathbf{v}_{p}\right|\right)$, respectively, as a functions of both wavelength $\lambda$ and the angle $\theta$ for $\bar{C}=100$ and $\alpha=0.002$. For $\lambda$ held constant the phase speed decreases monotonically as function of $\theta$ and, as expected, goes to zero as $\theta \rightarrow \pi / 2$. Comparison of Fig. 2a and b reveals only minor differences.

The wavelength dependency of the phase velocity gives rise to dispersion in the FS and the SSTREAM solutions. In these theories it is therefore somewhat misleading to interpret the phase velocity as the velocity by which surface disturbances propagate. The group velocity is a better measure of this velocity, and as shown in Gudmundsson (2003) the FS group velocity can be significantly different from the phase velocity and for some wavelengths even smaller than the material surface velocity.

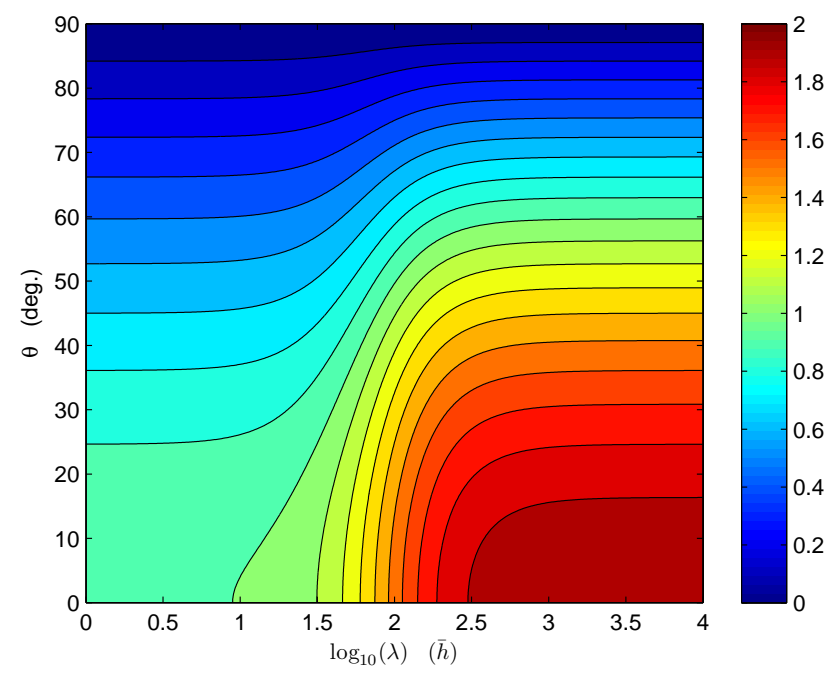

Fig. 2b. The shallow-ice-stream phase speed as a function of wavelength $\lambda$ and orientation $\theta$. As in Fig. 2a the mean surface slope is $\alpha=0.002$ and the slip ratio is $\bar{C}=100, n=m=1$, and the plot has been normalised with the non-dimensional surface velocity $\bar{u}=\bar{C}+1=101$ of the full-system solution.

One finds that in the SSTREAM approximation the $x$ and the $y$ components of the group velocity, $\left(u_{g}, v_{g}\right)$, are given by

$u_{g}=\bar{u}+\frac{\gamma+4\left(l^{2}-k^{2}\right) \bar{h} \eta \tau_{d}}{\left(\gamma+4 j^{2} \bar{h} \eta\right)^{2}}$,

and

$v_{g}=-\frac{8 k l \bar{h} \eta \tau_{d}}{\left(\gamma+4 j^{2} \bar{h} \eta\right)^{2}}$.

The $x$ component of the group velocity is shown in Fig. $1 \mathrm{~b}$ for $m=1$ as a function of wavelength for the same surface slope and slip ratio values as used in Fig. 1a. The figure shows that the SSTREAM expression for $u_{g}$ is a much better approximation to the FS solution than the one given by the SSHEET approximation. In fact the SSHEET group velocity does not look anything like the FS solution, whereas the SSTREAM solution traces the FS solution quite accurately (see Fig. 1b). Note also that, for the particular values of surface slope and slipperiness used in the Fig. 1b, the group velocity is smaller than the mean surface speed for wavelength between 8 to 50 mean ice thicknesses.

In Fig. 3 the relaxation time scale $\left(t_{r}\right)$ is plotted in dimensional units (years) as a function of wavelength for $\bar{C}=100$ and $\alpha=0.002$ and $m=1$. (Note that the relaxation time does not depend on the angle $\theta$.) As indicated by the figure, and direct inspection of the corresponding equations shows, the SSTREAM relaxation time scale closely approximates the FS relaxation time scale down to wavelengths of about 10 ice thicknesses. Of particular interest is the fact that in accordance with the full-system solution, but in contrast to the 


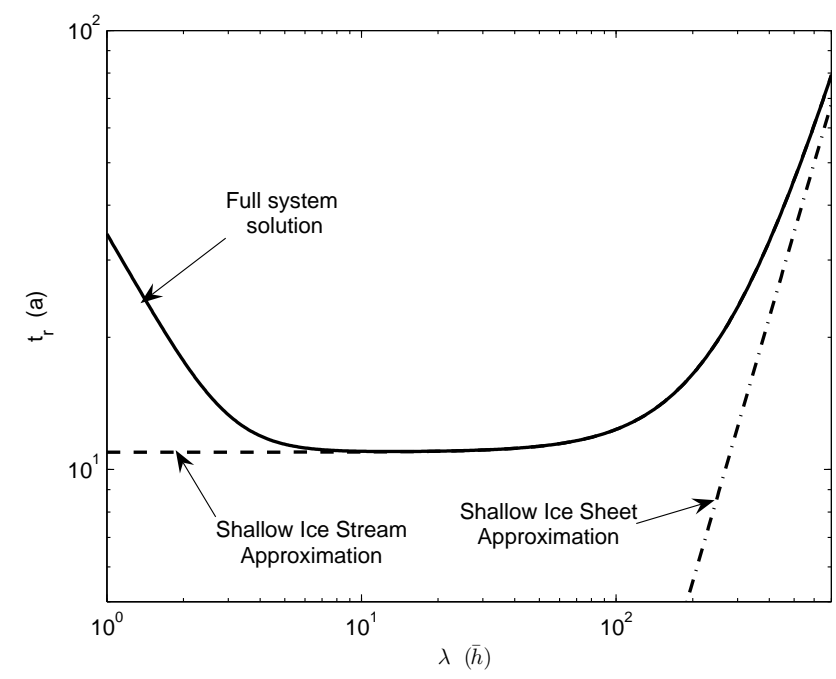

Fig. 3. The relaxation time scale $\left(t_{r}\right)$ as a function of wavelength $\lambda$. The wavelength is given in units of mean ice thickness $(\bar{h})$ and $t_{r}$ is given in years. The mean surface slope is $\alpha=0.002$, the slip ratio is $\bar{C}=999$, and $n=m=1$. For these values $t_{r}$ is on the order of 10 years for a fairly wide range of wavelengths. Lowering the slip ratio will reduce the value of $t_{r}$. It follows that ice streams will react to sudden changes in basal properties or surface profile by a characteristic time scale of a few years.

SSHEET approximation, there is a range of wavelengths over which the SSTREAM relaxation time scale is independent of $\lambda$ (see Fig. 3). The only qualitative aspect of $t_{r}$ not captured by the SSTREAM approximation is the increases in $t_{r}$ with $\lambda$ decreasing for $\lambda$ less than about ten ice thicknesses. For $\bar{C} \gg 1$ the expression for $t_{r}$ given by the SSTREAM solution is a much better approximation to $t_{r}$ than the one given by the SSHEET solution. For the particular set of parameters used in Fig. 3 both the SSTREAM and the FS solutions give, for the range $10 \bar{h}<\lambda<100 \bar{h}$, a constant value for $t_{r}$ on the order of ten years. The SSHEET solution gives, for the same range values, estimates of $t_{r}$ ranging from hours to days.

Of the two time scales $t_{r}$ and $t_{p}$ it can be argued that $t_{r}$ is the more important one. A surface wave will travel a distance equal to its wavelength in the time $2 \pi t_{p}$. In the same time its amplitude will decrease by the factor $e^{2 \pi t_{p} / t_{r}}$ (See Eq. 27). The ratio $2 \pi t_{p} / t_{r}$, thus, can be thought of as giving the relative importance of relaxation/diffusion to wave propagation. As pointed out by Jóhannesson (1992) this ratio is, for typical situations encountered in glaciology, usually larger than one. It follows that the time scale for local mass redistribution on glaciers and ice sheets is essentially given by $t_{r}$, and takes place much faster than the time scale $t_{p}$ would suggest. The close agreement between $t_{r}$ as given by the FS and the $t_{r}$ as calculated on basis of the SSTREAM approximation gives added confidence in the applicability of the SSTREAM approximation to situations typical of active ice streams.

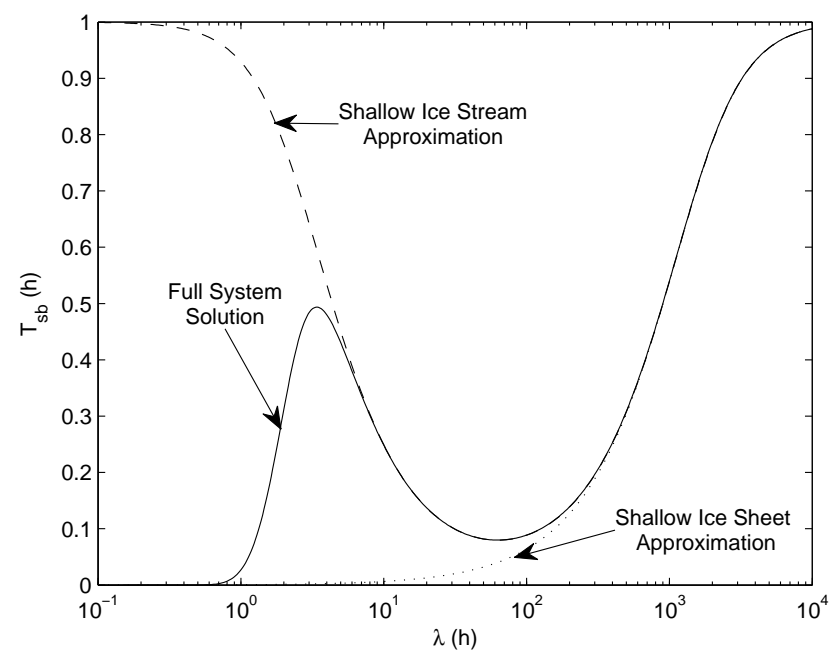

Fig. 4. Steady-state response of surface topography $(\Delta s)$ to a perturbation in bed topography $(\Delta b)$. The surface slope is 0.002 , the mean slip ratio $\bar{C}=100$, and $n=m=1$. Transfer functions based on the shallow-ice-stream approximation (dashed line, Eq. 20), the shallow-ice-sheet approximation (dotted line, Eq. 19 in Gudmundsson, 2003) and a full system solution (solid line, Eq. 75 in Gudmundsson, 2003) are shown.

\subsection{Bed topography perturbations}

Figure 4 shows the steady-state bedrock-to-surface transfer amplitude $\left(\left|T_{s b}\right|\right)$ as a function of wavelength. The figure shows the FS, the SSHEET, and the SSTREAM transfer amplitudes, respectively (based on Eqs. 82 and 26 in Gudmundsson, 2003, and Eq. 20). Surface slope $(\alpha=0.002)$ and slip ratio $(\bar{C}=100)$ values are typical for active ice streams. The three curves differ in a number of important ways.

The steady-state SSHEET transfer amplitude (dotted line in Fig. 4) is a strictly increasing function of wavelength and does not show the pronounced peak in bed-to-surface transfer for wavelengths from about 1 to 10 ice thicknesses seen in FS solution (solid line).

The SSTREAM solution (dashed line) overestimates the transfer at short wavelengths and gives a physically wrong limit of $\left|T_{s b}\right| \rightarrow 1$ for $\lambda \rightarrow 0$. Despite the incorrect limit for $\lambda \rightarrow 0$, the SSTREAM solution for $T_{s b}$ is, and this is generally the case for high slip ratios, a considerably better approximation to the FS solution than the corresponding SSHEET solution. For the particular set of values used in Fig. 4 the SSTREAM solution agrees within a few percent to the FS solution down to wavelengths of about eight ice thicknesses, while the SSHEET solution is only similarly accurate for wavelengths larger than about 100 ice thicknesses.

From Eq. (20) it follows that the steady-state SSTREAM transfer function for $l=0$ is

$T_{s b}=\frac{1}{1+\frac{\mathrm{i} k m \cot \alpha}{1+m\left(1+2 \bar{C} k^{2}\right)}}$, 


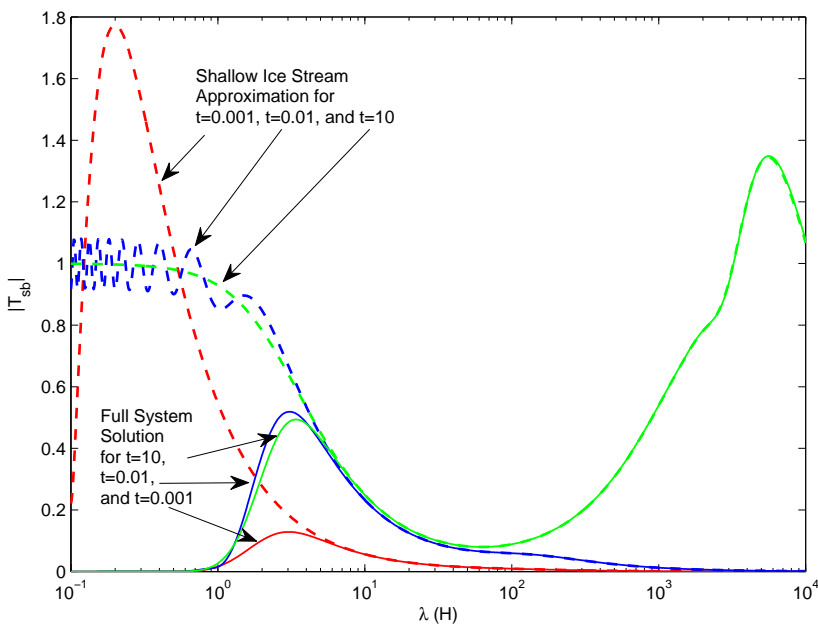

Fig. 5. Transient surface topography response to a sinusoidal perturbation in bed topography applied at $t=0$. Shown are the amplitude ratios between surface and bed topography $\left(\left|T_{s}\right|\right)$ as a function of wavelength for $\alpha=0.002, \theta=0, \bar{C}=100$, and $n=m=1$ for $t=0.001$ (red), $t=0.01$ (blue), and $t=10$ (green).

where dimensionless units have been used. Hence for $\theta=0$, $T_{s b} \rightarrow 1$ as $\bar{C} \rightarrow \infty$ irrespective of the values for surface slope $\alpha$, wavelength $\lambda$, and sliding law exponent $m$. The value of $\bar{C}=100$ used in Fig. 4 can hardly be considered very large for typical active ice streams, and if a value of $\bar{C}=1000$ is used together with typical surface slopes of about 0.002 to 0.004 , it follows that $\left|T_{s b}\right|$ is fairly close to unity for all wavelengths.

The minimum in the SSTREAM transfer amplitude given by Eq. (35) is reached for the wavelength

$\lambda_{x}=2 \pi \sqrt{\frac{2 \bar{C} m}{1+m}}$,

where $k \lambda_{x}=2 \pi$, and the minimum is given by

$$
\min _{k}\left|T_{s b}\right|=\frac{1}{\sqrt{1+\frac{m \cot ^{2} \alpha}{8 \bar{C}(1+m)}} .}
$$

For bed topography variations aligned transversely to the main flow direction, the steady-state ratio between surface and bed topography amplitudes is in the SSTREAM approximation always larger than that given by Eq. (37). The wavelength given by Eq. (36) does not depend on surface slope $\alpha$. As Fig. 4 suggests, the (global) minimum in SSTREAM transfer amplitude $\left(\left|T_{s b}\right|\right)$ closely approximates the corresponding (local) minimum of the FS transfer amplitude for $\bar{C} \gg 1$.

Examples for non-steady $T_{s b}$ amplitudes are shown in Fig. 5. The dashed lines in the figure are calculated using Eq. (20) and are based on the shallow-ice-stream approximation, using the additional small-amplitude assumption $\Delta b / \bar{h} \ll 1$. The solid lines are FS solutions calculated using Eq. (82) in Gudmundsson (2003). The times are given in non-dimensional units. These can be translated to dimensional units through multiplication with $\bar{h} / \bar{u}_{d}$, where $\bar{u}_{d}$ is the mean deformational velocity. As an example, for a $1000 \mathrm{~m}$ thick ice stream where the surface velocity is $1 \mathrm{~m} \mathrm{~d}^{-1}, t=0.001$ corresponds to about 3.3 months.

The figure shows the relatively slow increase in $\left|T_{s b}\right|$ with time for long wavelengths (larger than about 100 mean ice thicknesses) toward the steady-state long-wavelength limit of $\left|T_{s b}\right|=1$. The rate of increase toward the steady-state limit is determined by $t_{r}$ which, for long wavelengths, increases quadratically as a function of wavelength (see Eq. 17 and Fig. 3), hence the slow increases in $\left|T_{s b}\right|$ for long wavelengths. Over wavelengths less than about 5 mean ice thicknesses the SSTREAM relaxation time is smaller than the FS relaxation time (see Fig. 3). Consequently, over this range of wavelengths the SSTREAM amplitudes grow faster with time than the FS amplitudes. Another noticeable aspect of Fig. 5 is the oscillating behaviour of the transfer amplitudes with wavelength. These are caused by temporal fluctuations (kinematic oscillations) in $\left|T_{s b}\right|$ that are governed by the phase time scale $t_{p}$. As the figure shows, transient amplitudes can be larger than unity, and when this happens surface topography amplitudes are larger than the bed topography amplitudes. As follows from inspection of Eq. (20) and Eq. (82) in Gudmundsson (2003), kinematic oscillation are particularly pronounced for $0<t<t_{r}$ whenever $t_{p}<t_{r}$. The consequence can be an up to twofold increase in transfer amplitudes as compared to the corresponding steady-state limit. As mentioned above, the steady-state $\lambda \rightarrow 0$ limit of the SSTREAM $T_{s b}$ amplitude is unity, and for short wavelengths $(\lambda / \bar{h}<1)$ the transient value can be as large as two, whereas the correct value for these wavelengths (as given by the FS solution) is always close to zero.

The $T_{s b}$ transfer amplitudes in Figs. 4 and 5 are plotted as functions of longitudinal wavelength, that is for sinusoidal variations in bed topography aligned transversely to the main flow direction. This corresponds to the situation $\theta=0$. Figure $6 \mathrm{a}$ and $\mathrm{b}$ show the SSTREAM and the FS transfer amplitudes, respectively, as functions of both $\theta$ and $\lambda$. As Fig. 4 showed for $\theta=0$, the main difference between the SSTREAM and the FS $T_{s b}$ amplitudes is the shortwavelength limit. Irrespectively of $\theta$ the SSTREAM shortwavelength limit is always equal to unity, whereas the correct limit is zero. The only exception is for the $\theta=\pi / 2$ where both transfer functions are identically zero.

In Fig. 7a and b the $T_{u b}$ transfer amplitudes of, respectively, the SSTREAM and FS solutions are shown in a nondimensionalised form. A simple way of interpreting the numerical contour values is to think of them as ratios between mean deformational velocity and mean ice thickness $\left(\bar{u}_{d} / \bar{h}\right)$. In the figures, a slip ratio of 99 is used and the mean surface FS velocity is equal to $100 \bar{u}_{d}$. For example, a transfer amplitude equal to 50 implies that a sinusoidal bed topography perturbation with an amplitude corresponding to $10 \%$ 


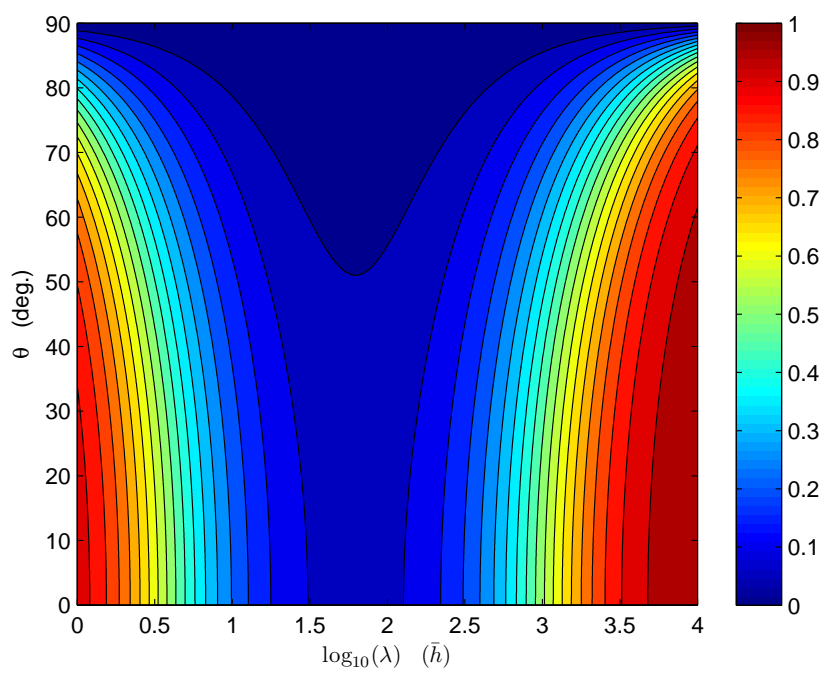

Fig. 6a. The SSTREAM amplitude ratio $\left(\left|T_{s b}\right|\right)$ between surface and bed topography (Eq. 20). Surface slope is 0.002 , the slip ratio $\bar{C}=99$, and $n=m=1 . \lambda$ is the wavelength of the sinusoidal bed topography perturbation and $\theta$ is the angle with respect to the $\mathrm{x}$ axis, with $\theta=0$ and $\theta=90$ corresponding to transverse and longitudinal undulations in bed topography, respectively.

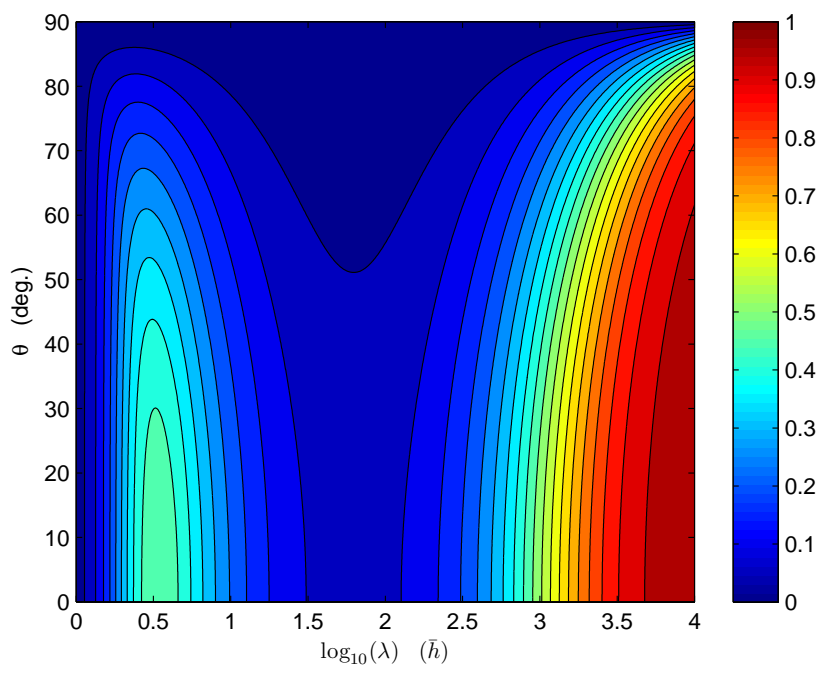

Fig. 6b. The FS amplitude ratio between surface and bed topography $\left(\left|T_{s b}\right|\right)$ from Eq. (75) in Gudmundsson (2003). The shape of the same transfer function for the same set of parameters based on the SSTREAM approximation is shown in Fig. 6a.

of the mean ice thickness produces a perturbation equal to $0.1 \times 50=5 \bar{u}_{d}$, or a $5 \%$ perturbation in surface velocity.

As the Fig. 7a and b suggest, and inspection of the corresponding analytical solutions confirms, the short wavelength limits of the SSTREAM and the FS $T_{u b}$ transfer amplitudes are both equal to zero. This is physically the correct limit and there is therefore no problem similar to that of the $T_{s b}$ transfer amplitude for $\lambda \rightarrow 0$. Quantitatively the $T_{u b}$ ampli-

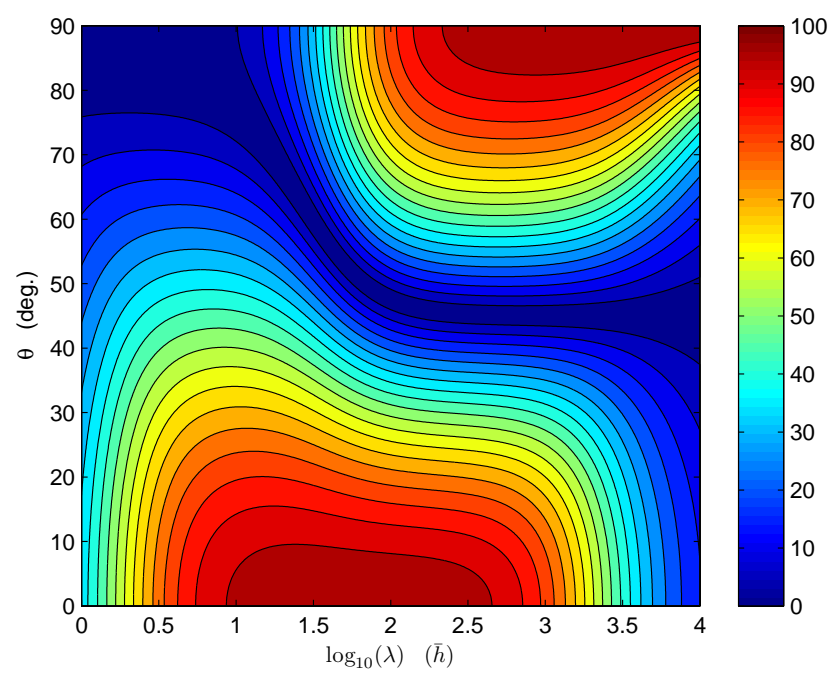

Fig. 7a. The steady-state amplitude ratio $\left(\left|T_{u b}\right|\right)$ between longitudinal surface velocity $(\Delta u)$ and bed topography $(\Delta b)$ in the shallowice-stream approximation as given by Eq. (23). Surface slope is 0.002 , the slip ratio is 99 , and $n=m=1$.

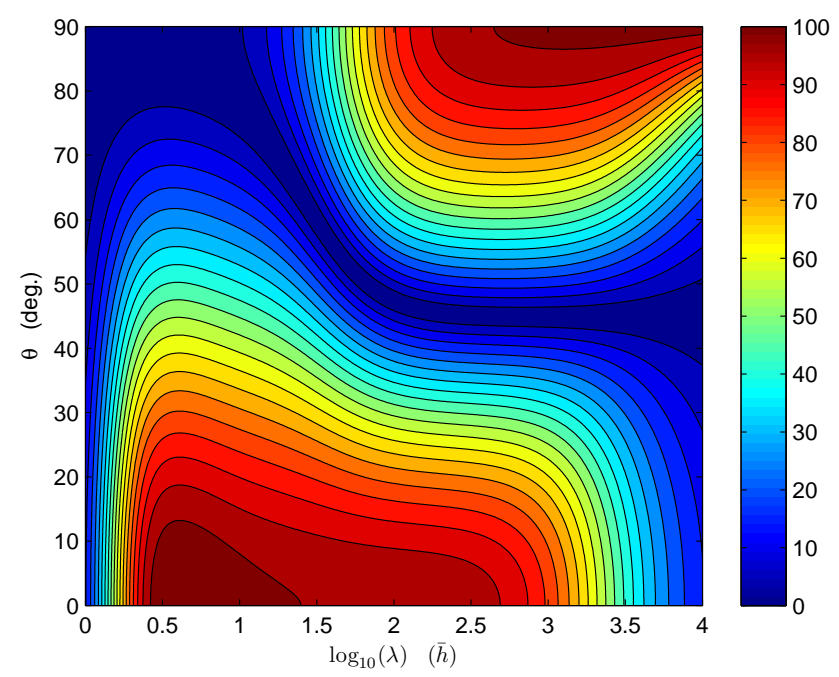

Fig. 7b. The steady-state amplitude ratio $\left(\left|T_{u b}\right|\right)$ between longitudinal surface velocity $(\Delta u)$ and bed topography $(\Delta b)$ (Eq. 75 in Gudmundsson, 2003). The shape of the same transfer function for the same set of parameters, but based on the shallow-ice-stream approximation, is shown in Fig. 7a.

tudes tend to be somewhat underestimated by the SSTREAM approximation when the shallow-ice condition $\lambda / \bar{h} \gg 1$ is not fulfilled.

For bed topography disturbances running along the flow $(k=0)$ the resulting perturbation in surface velocity is, irrespectively of $t$, given by

$T_{u b}=-\frac{2 m \bar{C}}{2+l^{2} m \bar{C}}$, 


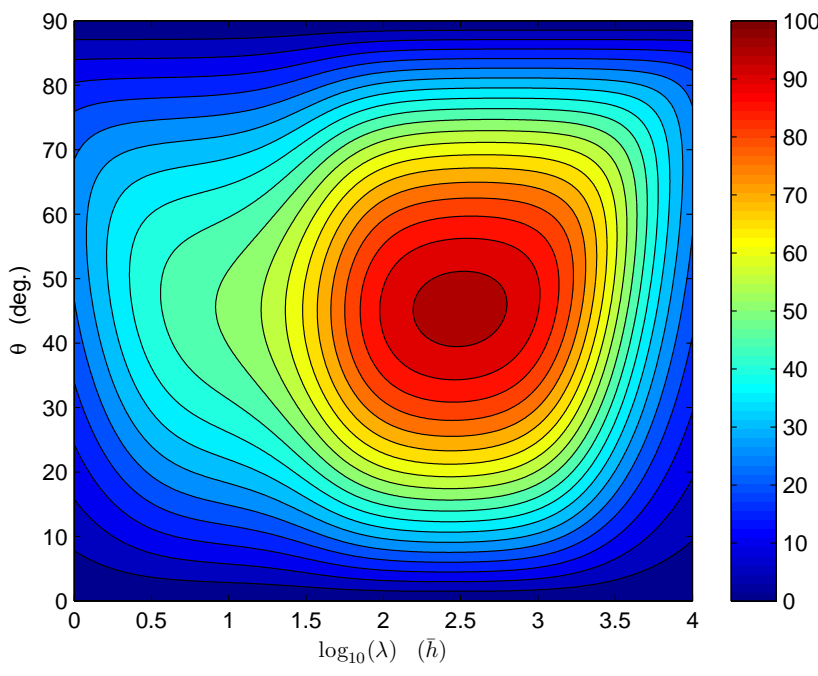

Fig. 8a. The steady-state amplitude ratio $\left(\left|T_{v b}\right|\right)$ between transverse velocity $(\Delta v)$ and bed topography $(\Delta b)$ in the shallow-ice-stream approximation (Eq. 24). Surface slope is 0.002 , the slip ratio is 99 and $n=m=1$.

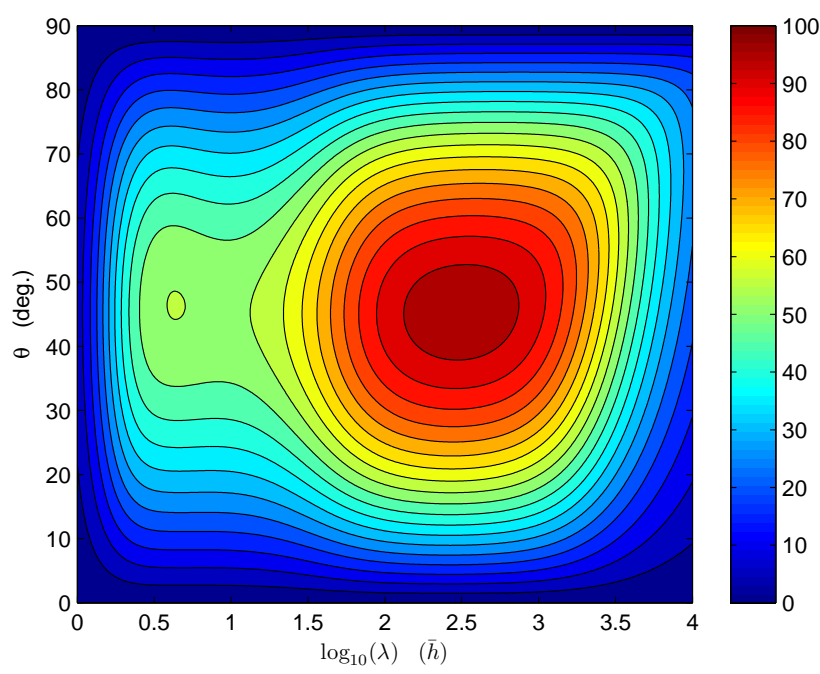

Fig. 8b. The steady-state amplitude ratio $\left(\left|T_{v b}\right|\right.$ between transverse velocity $(\Delta v)$ and bed topography $(\Delta b))$ from Eq. (75) in Gudmundsson (2003). The shape of the same transfer function for the same set of parameters, but based on the shallow-ice-stream approximation, is shown in Fig. 8a.

as can readily be derived from Eq. (23). The maximum value of $\left|T_{u b}\right|$ is reached for $k=0$ in the limit $\lambda \rightarrow \infty$. In the FS theory this limit is $\bar{C}+1$ which is the physically correct limit. In the SSTREAM theory this limit is, for $m=1$ where these results can be compared, equal to $\bar{C}$, the difference being due to the simple fact that the SSTREAM approximation ignores the internal ice deformation.

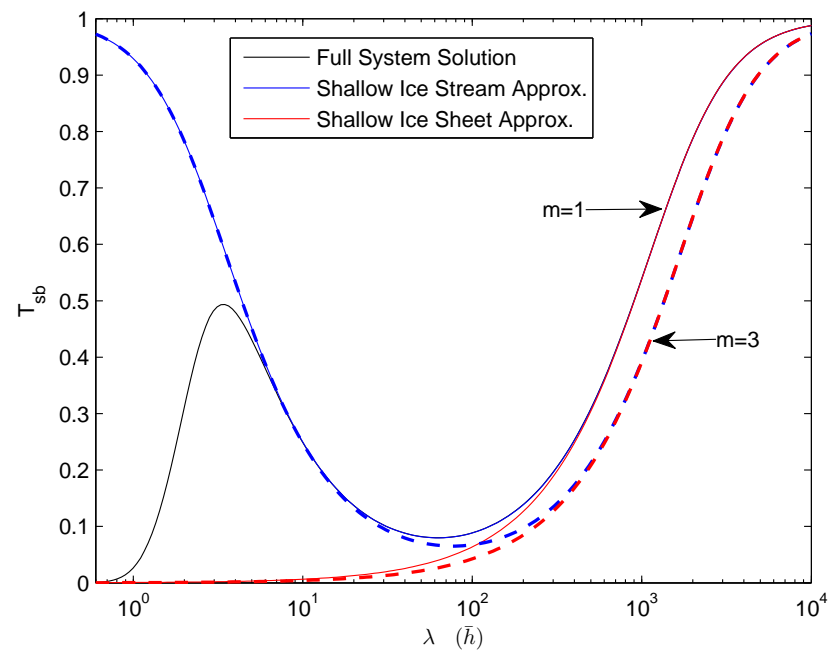

Fig. 9. Steady-state response of surface topography to a perturbation in bed topography for linear and non-linear sliding. All curves are for linear medium $(n=1)$. The solid lines are calculated for linear sliding $(m=1)$ and the dashed lines for non-linear sliding $(m=3)$. The red lines are SSHEET solutions, the blue ones are SSTREAM solutions, and the black line is a FS solution which is only available for $m=1$. Mean surface slope is 0.002 and slip ratio is equal to 100 .

For the transverse velocity amplitudes $\left(\left|T_{v b}\right|\right)$, shown in Fig. 8a and b, a qualitative difference between the FS and the SSTREAM solutions is found for $\theta$ close to $\pi / 4$. For $\theta=\pi / 4$ the SSTREAM transfer amplitude $\left|T_{v b}\right|$ has one local maximum as function of $\lambda$ but the FS solution two. Again the SSTREAM $T_{v b}$ amplitudes are somewhat underestimated when the shallow-ice condition $\lambda / \bar{h} \gg 1$ is not fulfilled. For the particular set of parameters using in Fig. $7 \mathrm{a}$ and $b$, and Fig. 8a and $\mathrm{b}$ the SSTREAM approximation underestimates the effects of bed perturbations on surface velocities for wavelengths less than about 10 ice thicknesses.

\subsubsection{Surface topography and non-linear sliding}

The discussion given above has mostly dealt with the $n=1$ and $m=1$ case. In Fig. 9 the SSHEET and the SSTREAM ratios between surface and bed topography amplitude $\left(\left|T_{s b}\right|\right)$ are plotted for $m=1$ and $m=3$ for $n=1$. In addition the FS ratio is shown for $m=1$. There are no analytical FS solutions known for $m \neq 1$. The SSTREAM ratios shown (blue lines in Fig. 9) follow from Eq. (20), the FS ratio (black line) from Eq. (82) in Gudmundsson (2003), and the SSHEET ratios (red lines) from Eq. (4.4.7) in Jóhannesson (1992).

As the Jóhannesson (1992) thesis is presumably not widely available I list here his SSHEET expression for the $T_{s b}$ transfer function. It is particularly simple and valid for any value of $n$ and $m$. It can be written in the form 


$$
T_{s b}=\frac{\mathrm{i}}{\mathrm{i}-\lambda^{\star} / \lambda},
$$

where

$\lambda^{\star}=\frac{\left(\frac{n(n+1)}{n+2}+m \bar{C}\right)}{n+1+\bar{C}(m+1)} \cot \alpha$,

is in units of mean ice thickness. The long and the short wavelength limits of SSHEET transfer amplitude as given by Eq. (39) are independent of $n$ and $m$, and the range of wavelengths where the transfer is, in absolute terms, significantly dependent on $n$ and $m$ is determined by the value of $\lambda^{\star}$. For moderate values of $n$ and high slip ratios $(\bar{C} \gg 1)$ we have

$\lambda^{\star} \approx \frac{m}{m+1} \cot \alpha$.

Hence, the surface slope is the most important parameter affecting the SSHEET bed-to-surface transfer characteristics for high slip ratios and the transfer is only moderately affected by the value of $m$.

One of the most interesting aspects of Fig. 9 is that the differences between the $m=1$ and the $m=3$ cases are only of any significance for wavelengths longer than about 100 ice thicknesses. This is the case for both the SSHEET and the SSTREAM solutions. By analysing the SSTREAM transfer function given by Eq. (20) it is found that the sensitivity of $\left|T_{s b}\right|$ to $m$ is small at both large and small wavelengths and decreases with decreasing slope. In particular, for wavelengths smaller than the wavelength for which the minimum in SSTREAM amplitude is reached (see Eq. 36), the transfer amplitudes are not significantly affected by the value of the sliding law exponent $m$.

No analytical FM solutions for $m>1$ are known, but a numerical study by Raymond and Gudmundsson (2005) showed that changing $m$ from 1 to 3 has almost no effect on FS transfer amplitudes for wavelengths smaller than $\lambda$ given by Eq. 36. The insensitivity of $T_{s b}$ to the value of the sliding law exponent $m$ for wavelengths smaller than the one given by Eq. (36) may, thus, well be a general feature of glacier flow. Schoof (2005) gives arguments suggesting that at high slip ratios the surface response becomes independent of the form of the sliding law. The results presented here, and those of Raymond and Gudmundsson (2005), show this only to be a good approximation for a limited set of wavelengths. The source of the discrepancy is not entirely clear, but possibly due to the implicit assumption in Schoof (2005) that shear stress is independent of short-scale perturbations in basal velocities (Eq. 3 in Schoof, 2005). On length scales on the order of one ice thickness or smaller Schoof (2005) results agree favourably with those presented here.

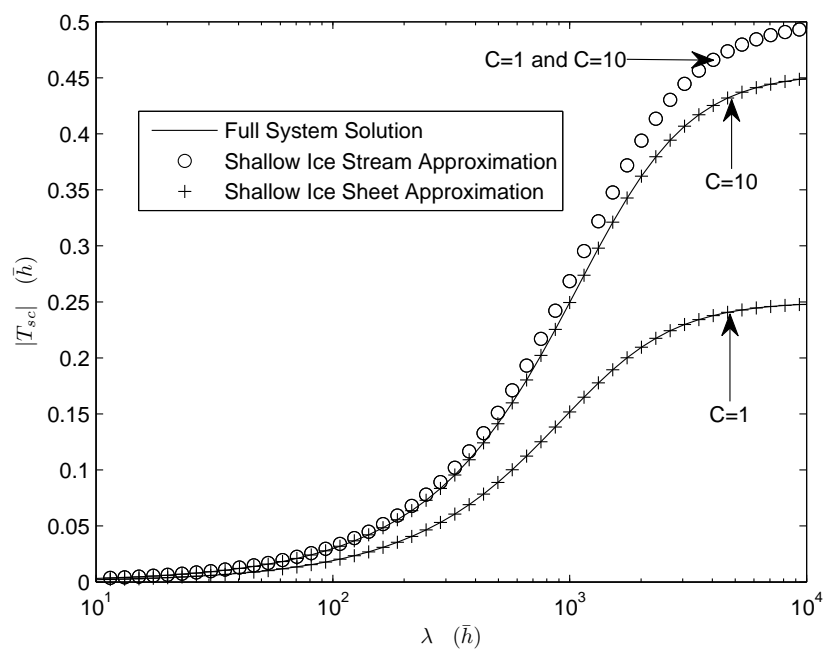

Fig. 10. Steady-state response of surface topography to a basal slipperiness perturbation. Shown are FS (solid line), SSTREAM (circles), and SSHEET (crosses) transfer amplitudes for both $\bar{C}=1$ and $\bar{C}=10$. In the plot the SSTREAM curves for $\bar{C}=1$ and $\bar{C}=10$ are too similar to be distinguished. The surface slope is 0.002 .

\subsection{Basal slipperiness perturbations}

We now consider the effects of spatial variations in basal slipperiness on surface fields. Basal slipperiness is here defined as the function $c(x, y)$ in the basal sliding law (see Eq. A5). The non-dimensional slipperiness is written using an upper case letter (i.e. $C(x, y)$ ). We have $C(x, y)=c(x, y) \tau_{d} / \bar{u}_{d}$, and write $C(x, y)=\bar{C}(1+\Delta C(x, y))$ where $\bar{C}$ is the spatially averaged slipperiness and $\Delta C(x, y)$ the (fractional) slipperiness perturbation introduced at $t=0$. The transfer functions listed in Appendix B give the relationships between surface fields and the basal slipperiness perturbation $\Delta C(x, y)$ in frequency space. We have, for example, $s(k, l, t)=T_{s c} \Delta C(k, l)$. Again our main focus here is on the differences between the corresponding SSHEET, SSTREAM and the FS solutions and therefore the discussion is mostly limited to the $m=1$ and $n=1$ case where small-amplitude analytical solutions to all of FS, SSHEET, and the SSTREAM problems are available.

Figure 10 shows basal-slipperiness to surface geometry transfer amplitudes $\left(T_{s c}\right)$ based on Eq. (B3) (circles) for the SSTREAM theory. For comparison the predictions of the FS (solid lines ) and the SSHEET (crosses) theories (Eqs. 83 and 27 in Gudmundsson, 2003) for the same parameter set are shown as well. Because the SSTREAM solution does not include the contribution of internal ice deformation to the forward surface velocity, the SSTREAM and the FS long wavelength limits for $\left|T_{s c}\right|$ are not equal. Both the FS and the SSHEET long wavelength limits $(\lambda \rightarrow \infty$ for $\theta=0)$ are equal to $\bar{C} /(2(1+\bar{C}))$. For the SSTREAM solution this limit is, on the other hand, equal to $1 / 2$ independently of $\bar{C}$ (see Eq. B7). For $\bar{C} \gg 1$ these two different expressions, of course, give numerically quite similar answers. 


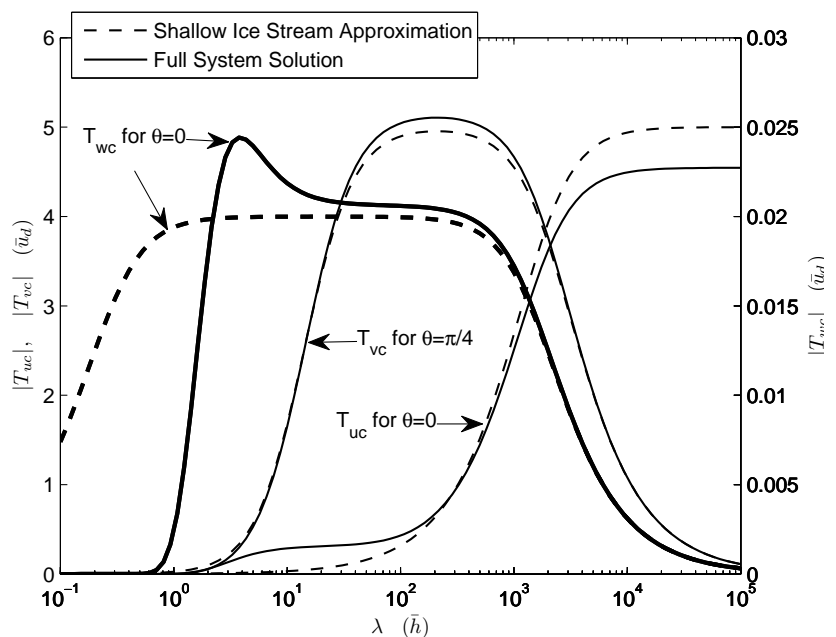

Fig. 11. Steady-state response of surface longitudinal $(u)$, transverse $(v)$, and vertical $(w)$ velocity components to a basal slipperiness perturbation. The surface slope is 0.002 and the slip ratio $\bar{C}=10$. The $T_{u c}$ and $T_{w c}$ amplitudes are calculated for slipperiness perturbations aligned transversely to the flow direction $(\theta=0)$. For $T_{v c}, \theta=45$ degrees. Of the two y axis the scale to the left is for the horizontal velocity components $\left(T_{u c}\right.$ and $\left.T_{w c}\right)$, and the one to the right is the scale for $T_{u c}$.

Figure 11 shows the velocity transfer amplitudes $\left|T_{u c}\right|$, $\left|T_{v c}\right|$, and $\left|T_{w c}\right|$. Shown are both the FS solutions (solid lines) and those based on the SSTREAM approximation (dashed lines). The SSTREAM solutions are given by Eqs. (B4), (B5) and (B6). The FS solutions can be found, or easily derived, from Gudmundsson (2003). The transfer functions are plotted in non-dimensional form. The scale for the transfer functions follows from the definitions $\Delta u=T_{u c} \Delta C, \Delta v=T_{v c} \Delta C$, where $\Delta C$ is the (fractional) slipperiness perturbation. Since the velocity is scaled with the mean deformational velocity $\bar{u}_{d}$ and $\Delta C$ has no dimensions, $\bar{u}_{d}$ is the scale for these transfer functions.

Generally speaking there is a good agreement between the velocity transfer functions of the FS and the SSTREAM solutions (see Fig. 11). However, there are also a number of significant differences. The SSTREAM long-wavelength limit of $T_{u c}$ is, for example, not the same as that of the FS theory. For $n=1$ and $m=1$, and $\theta=0$ we find that the SSTREAM solution gives

$$
\lim _{\lambda \rightarrow \infty} T_{u c}=\frac{1}{2} \bar{C}
$$

while the FS solution gives

$$
\lim _{\lambda \rightarrow \infty} T_{u c}=\frac{\bar{C}^{2}}{2(1+\bar{C})} \text {. }
$$

The effects of basal slipperiness perturbations are therefore somewhat overestimated for long-wavelengths, although for high slip ratios typical of active ice streams this error is small.

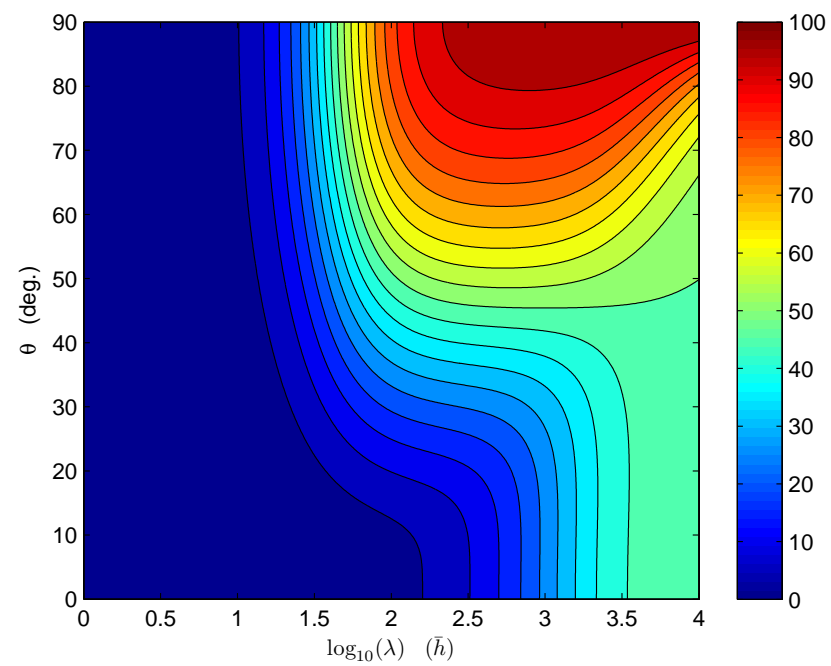

Fig. 12. Steady-state response of the surface longitudinal $(\Delta u)$ velocity component to a basal slipperiness perturbation in the shallowice-stream approximation (Eq. B4). The surface slope is 0.002 and the slip ratio $\bar{C}=99$.

Over wavelengths less than about 100 ice thicknesses the horizontal forward velocity component $(u)$ reacts weakly to basal slipperiness perturbations. In Fig. 11, for example, the FS $T_{u c}$ transfer amplitudes (solid line) are less than $5 \%$ of mean surface speed for this wavelength range. The SSTREAM approximation further underestimates this weak response (see Fig. 11). In comparison to the FS amplitude the SSTREAM $T_{w c}$ amplitudes are, on the other hand, too large (see Fig. 11). This difference is in most situations of no real concern, however.

The most conspicuous aspect of both the FS and the SSTREAM $T_{w c}$ transfer amplitudes is how small they are both in absolute terms and in comparison to $\left|T_{u c}\right|$ and $\left|T_{v c}\right|$. In fact the $\left|T_{w c}\right|$ amplitudes are so small that for active ice streams the vertical velocity component can be considered to be effectively insensitive to any spatial variations in basal slipperiness. As an example, for $\bar{C}=10$ and $\alpha=0.002$, which are the values used in Fig. 11, the maximum of the FS $T_{w c}$ amplitude is about 0.025 . The corresponding perturbation in the vertical surface velocity component is therefore $0.025 \bar{u}_{d}$ which for most active ice streams, where the surface velocity is a few hundred to a few thousand times larger than $\bar{u}_{d}$, is negligible in comparison to the mean horizontal velocity. In contrast, the maximum perturbation in longitudinal velocity $(u)$ is $\bar{C} / 2$ for $\bar{C} \gg 1$ or about $50 \%$ of the mean surface velocity, and as can be seen from Fig. 11 or direct inspection of Eq. (B5). Equally large perturbations in transverse velocity are possible for perturbations in basal slipperiness that are sufficiently misaligned with respect to the mean flow direction. 
The transfer amplitude $T_{u c}$ describing the response of the horizontal forward velocity component $(u)$ to a basal slipperiness perturbation is shown as a function of wavelength $(\lambda)$ and orientation $(\theta)$ in Fig. 12. This Figure should be compared to Fig. 7a giving the $u$ response to a bed topography perturbation for the same set of parameters. A noticeable difference between $T_{u b}$ and $T_{u c}$ amplitudes is the comparatively weak response of $u$ to perturbation in basal slipperiness that are aligned approximately transversely to the mean flow $(\theta<40$ degrees) for wavelengths less than about 100 ice thicknesses. However, for a given wavelength, the magnitude of the perturbation in $u$ caused by a perturbation in basal slipperiness increases sharply with $\theta$ (see Fig. 12). For a sinusoidal slipperiness perturbation closely aligned with the mean flow direction $(\theta>70$ degrees) the resulting perturbation in forward velocity can become a sizable fraction of the total perturbation in slipperiness $(\bar{C} \Delta C)$. For example, Fig. 12 shows that a sinusoidal basal slipperiness perturbation with wavelength $\lambda=100$ has almost negligible effect on $u$ if it is aligned transversely to the mean flow direction $(\theta=0)$, while the perturbation in $u$ is almost equal to the total perturbation in slipperiness for $\theta=90$ degrees.

In Appendix B the SSTREAM version of the $T_{u c}$ transfer function is derived. From Eq. (B4) we find that for perturbations in basal slipperiness that vary across the flow, i.e. $\theta=\pi / 2$ and $k=0$, the transfer function is

$$
T_{u c}=\frac{2 \bar{C}}{2+m l^{2} \bar{C}},
$$

for any $t$. Interestingly, as Eq. (41) shows, the response of the velocity to temporal changes in basal slipperiness perturbations aligned with the mean flow direction is instantaneous. Note furthermore that since $T_{u c}=\Delta u / \Delta C$ by definition, $T_{u c}=\bar{C}$ corresponds to a situation where the perturbation in surface velocity is equal, in non-dimensional units, to the (total) perturbation in basal slipperiness. This can be considered to represent a full transmission of basal slipperiness to the surface velocity field. As Eq. (41) shows, longitudinal basal slipperiness perturbations $(\theta=\pi / 2)$ have, in this sense, full effect on the forward surface velocity component (u) in the limit $\lambda \rightarrow \infty$. Note also that for $k=0$ the limit of $T_{u c}$ when $\lambda \rightarrow \infty$ is independent of the sliding law exponent $m$.

For $\theta=0$, i.e. where basal slipperiness varies in direction parallel to the flow direction but not across the flow, the situation is very different from that for $\theta=\pi / 2$. From B4 given in Appendix B we find that in dimensionless units

$T_{u c}=\frac{\bar{C}}{1+m\left(1+2 k^{2} \bar{C}+\mathrm{i} k m \cot \alpha\right)}$,

for $l=0$. In particular

$$
\lim _{k \rightarrow 0} T_{u c}=\frac{\bar{C}}{1+m},
$$

showing that the long wavelength limit for $\theta=0$ is always smaller than that for $\theta=\pi / 2$ and, furthermore, goes to zero with increasing $m$.

Raymond (1996) calculated the effects of longitudinal perturbations in slipperiness on surface velocity on ice streams $(\theta=\pi / 2)$. In the dimensionless notation used here his result (Eq. 25a in Raymond, 1996) is

$T_{u c}(k=0, l)=\frac{2 \bar{C}}{2+\bar{C} l \tanh l}$.

From Gudmundsson (2003) one finds after some calculations that the FS expression is

$T_{u c}(k=0, l)=\frac{2 \bar{C}}{2 \cosh l+\bar{C} l \sinh l}$.

Raymond (1996) considered the case $\bar{C} \gg 1$ and consequently the form of the basal boundary conditions used by him is slightly different from the one used in Gudmundsson (2003). This explains the difference between Eqs. (44) and (45).

In contrast to Eqs. (44) and (45), Eq. (41) is not limited to linear sliding law but valid for any positive value of $m$. Where Eqs. (41), (44), and (45) can be compared, i.e. for $m=1$ the difference between these three expressions is in most cases small. In particular, all expressions give the same limits for $l \rightarrow 0, l \rightarrow+\infty$ for $\bar{C}$ fixed, and $\bar{C} \rightarrow 0$ for $l$ fixed. For $\bar{C} \rightarrow+\infty$ the limits are not identical but for $l \ll 1$ the difference is small.

\subsection{Flow over Gaussian peak: the ISMIP-HOM Experi- ment $\mathrm{F}$}

As an illustration of differences between the full-system and the shallow-ice-stream solutions Fig. 13a and b show an example of the surface response to a Gaussian-shaped bedrock protuberance calculated using both the FS and the SSTREAM transfer functions. The parameters of the example are motivated by the definition of the Benchmark Experiment $\mathrm{F}$ for higher-order ice sheet models of the ongoing model intercomparision project ISMIP-HOM (see http: //homepages.ulb.ac.be/ fpattyn/ismip/). Of the mean surface velocity, half is due to internal ice deformation and the other half to basal sliding $(\bar{C}=1)$. The flow is down an uniformly inclined plane with a mean slope of 3 degrees. The bedrock perturbation is a Gaussian shaped peak situated at $(x, y)=(0,0)$. The peak has a width of $10 \bar{h}$ and amplitude of $0.1 \bar{h}$. Periodic boundary conditions are used with a periodicity of $400 \bar{h}$ in both $x$ and $y$ directions.

If either the slip ratio is not large compared to unity, or the horizontal scale of interest is not large compared to mean ice thickness, significant deviations between the SSTREAM and the FS solutions can be expected. In the experiment the slip ratio does not fulfil the condition $\bar{C} \gg 1$ and it comes as no surprise that there are some differences between the upper (SSTREAM solution) and the lower (FS solution) halves of Fig. 13a and b. However, if anything, the performance of the 


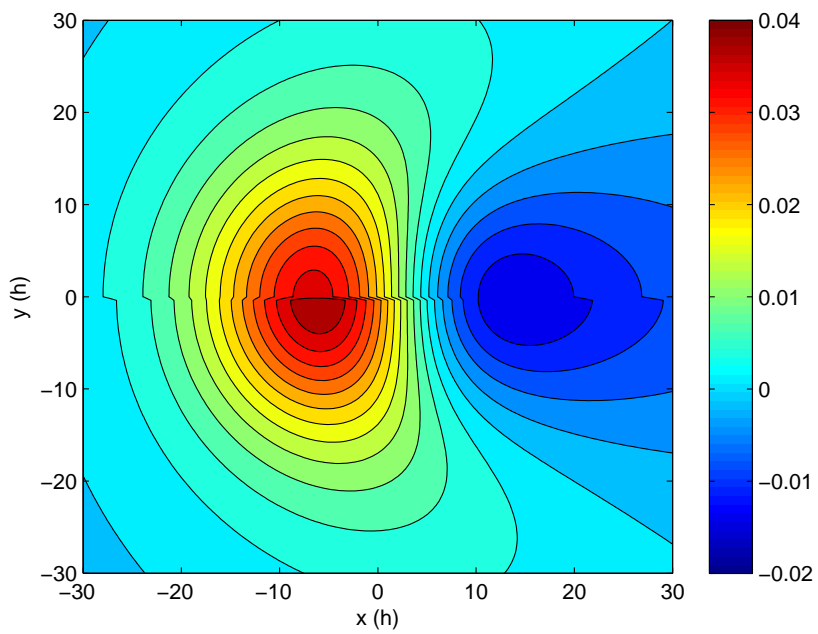

Fig. 13a. Surface topography response to a flow over a Gaussianshaped bedrock disturbance as given by a FS (lower half of figure) and a SSTREAM solution (upper half of figure). The mean flow direction is from left to right. Surface slope is 3 degrees and mean basal velocity equal to mean deformational velocity $(\bar{C}=1)$. The spatial unit is one mean ice thickness $(\bar{h})$. The Gaussian-shaped bedrock disturbance has a width of $10 \bar{h}$ and it's amplitude is $0.1 \bar{h}$. The problem definition is symmetrical about the $\mathrm{x}$ axis $(y=0)$ and any deviations in the figure from this symmetry are due to differences in the FS and the SSTREAM solutions.

SSTREAM solutions seems surprisingly good. There are, for example, only fairly small differences seen in the perturbed surface topography (Fig. 13a). The amplitude of the FS surface topography perturbation (lower half of Fig. 13a) is a bit larger than that of the SSTREAM one (upper half of Fig. 13a) but otherwise the surface shapes are in qualitatively terms the same.

The FS velocity perturbations are generally larger than the those of the SSTREAM approximation (see Fig. 13b). This is partly due to the simple fact that the mean SSTREAM velocity is equal to 1 while the mean FS velocity is twice as large. One could argue that the mean slip ratio in the SSTREAM theory should be redefined to give the same mean surface velocity as the FS solution. In the experiment this would imply using $\bar{C}=2$ when calculating the SSTREAM solutions. When this is done, the differences between the upper and the lower halves in Fig. 13b become considerably smaller and the overall magnitude more similar. Irrespectively of which value of $\bar{C}$ is used, the FS velocity solution has a more detailed short-scale structure. This aspect of the solution can be understood by considering the corresponding transfer functions directly. Comparison of Fig. 7a with b, and Fig. 8a with $b$ (despite the parameters used in these figures being different from Experiment F) also illustrates the fact that the FS velocity transfer amplitudes are, for short to intermediate wavelengths, generally larger than those of the SSTREAM theory.

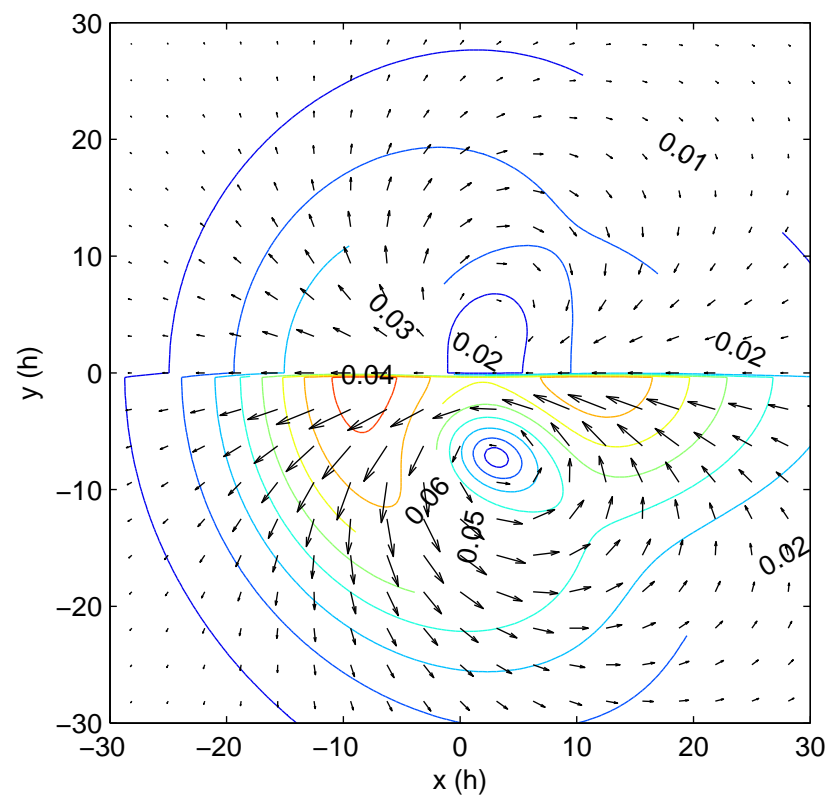

Fig. 13b. Response in surface velocity to a Gaussian-shaped bedrock perturbation. All parameters are equal to those in Fig. 13a. The contour lines give horizontal speed and the vectors the horizontal velocities. The velocity unit is mean-deformational velocity $\left(\bar{u}_{d}\right)$. The slip ratio is equal to one, and the mean surface velocity is $2 \bar{u}_{d}$. The upper half of the figure is the SSTREAM solution and the lower half the corresponding FS solution.

If the width of the Gaussian peak is decreased from $10 \bar{h}$ to, $1 \bar{h}$ and the slip ratio increased to 100 the differences in the calculated FS and the SSTREAM surface response become more pronounced. The amplitude of the SSTREAM topography perturbation is then much larger than that of the FS solution, and in fact quite similar to the shape of the bedrock perturbation itself. This aspect of the SSTREAM solution was discussed above and is caused by the fact that the SSTREAM $T_{s b}$ amplitude is close to unity for small wavelengths (see also Fig. 6a and b).

\section{Summary and conclusions}

As expected the comparison between the analytical FS and SSTREAM solutions shows that the SSTREAM approximation is highly accurate for long wavelengths $(\lambda / \bar{h} \gg 1)$ and high slip ratios $(\bar{C} \gg 1)$. The SSTREAM approximation is in these circumstances a much better approximation to the FS solutions than the SSHEET approximation. However, somewhat disappointingly, when these conditions are not fulfilled the SSTREAM approximation is not just inaccurate but gives rise to some physically unrealistic results. In particular, the ratio between surface and bed topography is overestimated. Whereas the correct ratio is close to zero for $\lambda \ll \bar{h}$ the SSTREAM gives a ratio close to unity. The SSHEET 
theory, which also is inaccurate for $\lambda \ll \bar{h}$, fails in this respect in a more physically realistic manner by underestimating the ratio and giving the correct limit of zero as $\lambda \rightarrow 0$. A related somewhat less than satisfying aspect of the $\left|T_{s b}\right|$ SSTREAM transfer amplitudes is the absence of the local maxima in the $T_{s b}$ amplitudes as function of wavelengths seen in the FS solution.

The physically wrong limit of the SSTREAM $T_{s b}$ amplitude for $\lambda \rightarrow 0$ is of some concern. It has, among other things, implications for surface-to-bed inversion. Because this $T_{s b}$ transfer amplitude limit is equal to unity, small scale surface undulations $(\lambda<\bar{h})$ do not get amplified through a direct inversion and the effects of surface data errors might be underestimated. There is also some danger of the spatial resolving power of an SSTREAM surface-to-bed inversion to be overestimated. Further research into these issues is needed before firm quantitative statements can be made.

The only previous work dealing with the effects of smallamplitude perturbations in boundary data on solutions of the SSTREAM theory is the numerical study of Hindmarsh (2004). He calculates $T_{s b}$ transfer amplitudes and both the $t_{r}$ and the $t_{p}$ timescales for linear and non-linear medium. The L1L1 approximation used by Hindmarsh (2004) is an improved version of the SSTREAM theory discussed here which includes the contribution of internal ice deformation to the velocity. For high slip ratios the L1L1 approximation is effectively equal to the SSTREAM approximation. Fig. 5 in Hindmarsh (2004) calculated for $n=3$ shows the same general features of transfer the amplitude $T_{s b}$ and the times scales $t_{p}$ and $t_{r}$ for non-linear rheology as found in the analytical solutions given here valid for $n=1$ and $m>0$. In particular the $\left|T_{s b}\right| \rightarrow 1$ limit for $\lambda \rightarrow 0$ is also found by Hindmarsh (2004) for non-linear medium (Fig. 5c). The relative insensitivity of the $t_{r}$ time scale to wavelengths for high slip ratios, and the chance in phase speed from $\bar{u}_{b}$ to $(m+1) \bar{u}_{b}$ with increasing wavelength is also seen (Fig. 5a and b in Hindmarsh, 2004).

The SSTREAM solutions are much better approximations to the FS solutions than the SSHEET solutions whenever $\bar{C} \gg 1$ and $\lambda / \bar{h} \gg 1$. For slip ratios typical of active ice streams the SSHEET solution underestimates the relaxation time scale by several order of magnitude. The SSTREAM solution for $t_{r}$ is, on the other hand, almost equal to the corresponding FS solution down to wavelengths of about $10 \bar{h}$. The SSTREAM solution gives a finite number for $t_{r}$ in the limit $\lambda \rightarrow 0$ (in dimensional units the limit is $4 c \eta \tan \alpha / u$ ) whereas the same limit for SSHEET theory is zero. It follows that once short wavelengths are present in an SSTREAM model they do not automatically decay away as they do in an SSHEET model.

Comparing the analytical solutions of the SSTREAM and the SSHEET approximations with the FS solutions presented here and in Gudmundsson (2003) provides a quick and an easy way of assessing the applicability of these two commonly used approximations to a particular situation. Results presented by Raymond and Gudmundsson (2005) on smallamplitude response for non-linear medium in the FS theory, and by Hindmarsh (2004) on numerical approximations to the Stokes equation for both linear and non-linear medium, suggest that this approach remains useful even when the rheology of the medium is non-linear. In particular, in cases where the analytical solutions reveal significant differences between the SSTREAM or the SSHEET approximations as compared to the FS solutions, it is improbable that inclusion of non-linear rheology will reduce these differences.

\section{Appendix A}

\section{Shallow-ice-stream scalings}

We consider the case of an ice stream with horizontal length scale $[x]$ and vertical length scale $[z]$ where the shallow-ice approximation $[z] /[x]=\delta \ll 1$ holds, and write

$(x, y, z)=[x]\left(x^{*}, y^{*}, \delta z^{*}\right)$.

where the asterisks denote scaled dimensionless variables. For the mass conservation equation $\left(v_{i, i}=0\right)$ to be invariant we scale the velocity as

$(u, v, w)=[u]\left(u^{*}, v^{*}, \delta w\right)$.

If we furthermore require the kinematic boundary condition at the surface

$\partial_{t} s+u \partial_{x} s+v \partial_{y} s-w=a$,

where $s$ is the surface to be invariant under the scalings we must have

$a=\delta[u] a^{*}$,

where $a$ is the accumulation rate. Thus the scale for $a$ is $[a]=\delta[u]=[w]$, which seems reasonable as we can expect the vertical velocity to scale with accumulation rate for small surface slopes. We also find using the same invariant requirement of the kinematic boundary condition of the surface that the time must be scaled as

$t=[x][u]^{-1} t^{*}$.

As a scale for the stress we use

$[\sigma]=A^{-1 / n}([a] /[z])^{1 / n}$,

which is motivated by the expectation $\dot{\epsilon}_{x x} \sim a / H$ and Glen's flow law

$\tau_{i j}=A^{-1 / n} \dot{\epsilon}^{(1-n) / n} \dot{\epsilon}_{i j}$,

where $\dot{\epsilon}$ is the effective strain rate defined through $\dot{\epsilon}$ : $=\left(\dot{\epsilon}_{i j} \dot{\epsilon}_{i j} / 2\right)^{1 / 2}$ and $\tau_{i j}$ are the deviatoric stress components. 
We are considering a situation where the vertical shear components are small compared to all other stress components. A set of scalings which reflects this situation is

$$
\begin{aligned}
& \left(\sigma_{x x}, \sigma_{y y}, \sigma_{z z}, \sigma_{x y}, \sigma_{x z}, \sigma_{y z}\right) \\
& =[\sigma]\left(\sigma_{x x}^{*}, \sigma_{y y}^{*}, \sigma_{z z}^{*}, \sigma_{x y}^{*}, \delta \sigma_{x z}^{*}, \delta \sigma_{y z}^{*}\right) .
\end{aligned}
$$

Same scale is used for the pressure, that is $p=[\sigma] p^{*}$,

The analysis is done in a coordinate system which is tilted forward in $x$ direction by the angle $\alpha$. The scaled momentum equations are

$$
\begin{aligned}
\partial_{x^{*}} \sigma_{x x}^{*}+\partial_{y^{*}} \sigma_{x y}^{*}+\partial_{z^{*}} \sigma_{x z}^{*} & =-\delta^{-1} \bar{\tau}[\sigma]^{-1}, \\
\partial_{x *} \sigma_{x y}^{*}+\partial_{y^{*}} \sigma_{y y}^{*}+\partial_{z^{*}} \sigma_{y z}^{*} & =0, \\
\delta^{2} \partial_{x^{*}} \sigma_{x z}^{*}+\delta^{2} \partial_{y *} \sigma_{y z}^{*}+\partial_{z^{*}} \sigma_{z z}^{*} & =\bar{\tau}[\sigma]^{-1} \cot \alpha,
\end{aligned}
$$

where $\bar{\tau}:=[z] \rho g \sin \alpha$. We obtain a consistent set of equations for $\alpha=O(\delta)$. The slope $\alpha$ is then no longer just an arbitrary tilt angel and $\bar{\tau}$ can be interpreted as a scale for the basal shear stress. From the scalings (A1) we then have $[\bar{\tau}]=\delta[\sigma]$, and it follows that the two non-zero terms on the right hand side of the system of Eqs. (A2) to (A4) are of order unity. Note that only terms of order $\delta^{2}$ are being dropped from the momentum equations.

We write the basal sliding law on the form

$\mathbf{u}_{b}=c(x, y)\left|\mathbf{T}_{b}\right|^{m-1} \mathbf{T}_{b}$,

where $\mathbf{T}_{b}$ is the basal stress vector given by $\mathbf{T}_{b}=\sigma \hat{\mathbf{n}}-\left(\hat{\mathbf{n}}^{T}\right.$. $\sigma \hat{\mathbf{n}}) \hat{\mathbf{n}}$, with $\hat{\mathbf{n}}$ being a unit normal vector to the bed pointing into the ice, and $\mathbf{u}_{b}$ is the basal sliding velocity given by $\mathbf{u}_{b}=$ $\mathbf{v}-\left(\hat{\mathbf{n}}^{T} \cdot \mathbf{v}\right) \hat{\mathbf{n}}$. The function $c(x, y)$ is referred to as the basal slipperiness. We find that components of the scaled basal stress vector $\left(\mathbf{T}_{b}^{*}\right)$ are given by

$$
\begin{aligned}
T_{b x}^{*}= & \delta \partial_{x}^{*} b^{*}\left(\sigma_{z z}^{*}-\sigma_{x x}^{*}\right)-\delta \partial_{y}^{*} b^{*} \sigma_{x y}^{*}+\delta \sigma_{x z}^{*}+O\left(\delta^{3}\right), \\
T_{b y}^{*}= & \delta \partial_{y}^{*} b^{*}\left(\sigma_{z z}^{*}-\sigma_{y y}^{*}\right)-\delta \partial_{x}^{*} b^{*} \sigma_{x y}^{*}+\delta \sigma_{y z}^{*}+O\left(\delta^{3}\right), \\
T_{b z}^{*}= & \delta^{2}\left(\left(\sigma_{z z}^{*}-\sigma_{x x}^{*}\right)\left(\partial_{x}^{*} b^{*}\right)^{2}+\left(\sigma_{z z}^{*}-\sigma_{y y}^{*}\right)\left(\partial_{y}^{*} b^{*}\right)^{2}\right. \\
& \left.-2 \sigma_{x y}^{*} \partial_{x}^{*} b^{*} \partial_{y}^{*} b^{*}+\sigma_{x z}^{*} \partial_{x}^{*} b^{*}+\sigma_{y z}^{*} \partial_{y}^{*} b^{*}\right)+O\left(\delta^{4}\right) .
\end{aligned}
$$

Thus $T_{b x}^{*}$ and $T_{b y}^{*}$ are $O(\delta)$ while $u_{b}^{*}$ and $v_{b}^{*}$ are $O(1)$, hence

$c[\sigma]^{m}[u]^{-1}=O\left(\delta^{-m}\right)$.

Note that since $[\bar{\tau}]=\delta[\sigma]$, we can also write Eq. (A9) as $c[\bar{\tau}]^{m}[u]^{-1} \delta^{-m}=O\left(\delta^{-m}\right)$ from which it follows that $c[\bar{\tau}]^{m}[u]^{-1}=O(1)$, as expected.

Using $\dot{\epsilon}_{x z}=A \tau^{n-1} \sigma_{x z}$ one finds that $\partial_{z} u=O\left(\delta^{2}\right)$, or smaller. Thus, to second order the horizontal velocity components $u$ and $v$ are independent of $z$. Differentiating the incompressibility equation $v_{i, i}=0$ with respect to $z$ it then follows that $\dot{\epsilon}_{z z}$ is independent of depth as well and that $w$ varies linearly with depth. A further consequence is that since the sliding velocity is of order unity and the deformational velocity $u_{d}=O\left(\delta^{2}\right)$ the slip ratio $\bar{u}_{b} / \bar{u}_{d}=O\left(\delta^{-2}\right)$. Note that in both the field equations and all boundary conditions all first order terms are identically equal to zero. The theory is consequently correct to second order in $\delta$.

Only collecting zeroth-order terms followed by vertical integration over depth and some simple manipulation leads to the following two coupled differential equations for the horizontal velocity components $u$ and $v$

$$
\begin{aligned}
& \partial_{x}\left(4 h \eta \partial_{x} u+2 h \eta \partial_{y} v\right)+\partial_{y}\left(h \eta\left(\partial_{x} v+\partial_{y} u\right)\right)-(u / c)^{1 / m} \\
& \quad=\rho g h \partial_{x} s \cos \alpha-\rho g h \sin \alpha, \\
& \partial_{y}\left(4 h \eta \partial_{y} v+2 h \eta \partial_{x} u\right)+\partial_{x}\left(h \eta\left(\partial_{y} u+\partial_{x} v\right)\right)-(v / c)^{1 / m} \\
& \quad=\rho g h \partial_{y} S \cos \alpha .
\end{aligned}
$$

Note that we have now gone back to the dimensional variables. The quantity $\eta$ is the effective viscosity defined through $\tau_{i j}=2 \eta \dot{\epsilon}_{i j}$. For Glen's flow law the effective strain rate is given by

$\dot{\epsilon}=\sqrt{\left(\partial_{x} u\right)^{2}+\left(\partial_{y} v\right)^{2}+\left(\partial_{y} u+\partial_{x} v\right)^{2} / 4+\partial_{x} u \partial_{y} v}$,

an expression that is correct to second order.

\section{Appendix B}

\section{Response of flow to basal slipperiness perturbations}

We consider the response to small perturbation in basal slipperiness. Writing $h=\bar{h}+\Delta s, s=\bar{s}+\Delta s, b=\bar{b}, u=\bar{u}+\Delta u$, $v=\Delta v, w=\Delta w$, and $c=\bar{c}(1+\Delta c)$ where $\Delta c$ is the fractional slipperiness, and inserting into (1) and (2) gives again the zeroth-order solution (3). The first-order field equations are

$$
\begin{gathered}
4 \eta \bar{h} \partial_{x x}^{2} \Delta u+3 \eta \bar{h} \partial_{x y}^{2} \Delta v+\eta \bar{h} \partial_{y y}^{2} \Delta u-\gamma \Delta u \\
=\rho g \bar{h} \cos \alpha \partial_{x} \Delta s-\rho g \sin \alpha \Delta s-\gamma \bar{u} \Delta c,
\end{gathered}
$$

and

$$
\begin{aligned}
& 4 \eta \bar{h} \partial_{y y}^{2} \Delta v+3 \eta \bar{h} \partial_{x y}^{2} \Delta u+\eta \bar{h} \partial_{x x}^{2} \Delta v-\gamma \Delta v \\
& =\rho g \bar{h} \cos \alpha \partial_{y} \Delta s .
\end{aligned}
$$

Fourier and Laplace transforming these equations and solving together with Eqs. (12) and (13), followed by an inverse Laplace transform gives

$$
\begin{aligned}
& T_{s c}=\frac{i k \bar{h} \bar{u} \gamma}{p \xi}\left(e^{p t}-1\right), \\
& T_{u c}=\frac{\gamma \bar{u}\left(\left(e^{p t}-1\right)\left(l^{2} \tau_{d} \bar{h} \cot \alpha-\mathrm{i} k \bar{u}\right)+e^{p t} p \phi\right)}{p \xi(\gamma+v)}, \\
& T_{v c}=\frac{k l \gamma \bar{u} \bar{h}\left(\left(1-e^{p t}\right)\left(\tau_{d} \cot \alpha-3 \mathrm{i} k \bar{u} \eta\right)-3 e^{p t} p \eta\right)}{p \xi(\gamma+v)},
\end{aligned}
$$

and

$$
T_{w c}=\frac{k \bar{h} \bar{u} \gamma\left(e^{p t}(\mathrm{i} p+k \bar{u})-k \bar{u}\right)}{p \xi},
$$


where $p$ is given by Eq. (15) and as before the abbreviations

$$
\begin{aligned}
\gamma & =\frac{\tau_{d}^{1-m}}{m \bar{c}}, \\
\tau_{d} & =\rho g \bar{h} \sin \alpha, \\
\nu & =\gamma+\bar{h} j^{2} \eta, \\
\xi & =\gamma+4 \bar{h} j^{2} \eta, \\
\phi & =\gamma+\bar{h}\left(k^{2}+4 l^{2}\right) \eta,
\end{aligned}
$$

have been used.

In some limiting cases these expressions obtain much simpler forms. In dimensionless units the steady-state form of the $T_{s c}$ transfer function is, for example

$$
T_{s c}=-\frac{k}{k+m\left(k\left(1+2 j^{2} \bar{C}\right)+i j^{2} \cot \alpha\right)} .
$$

$T_{s c}$ is a monotonically decreasing function of both $k$ and $m$. We find that

$\lim _{k \rightarrow 0} T_{s c}=\frac{1}{1+m}$,

for $l=0$ showing that in the long wavelength limit the SSTREAM surface topography response to basal perturbations is independent of mean slipperiness $\bar{C}$ but not of the sliding law exponent.

Acknowledgements. I thank R. Arthern, R. C. A. Hindmarsh, C. Martin and D. Vaughan for valuable discussions on early drafts of this manuscript. I'm grateful for helpful suggestions from the reviewer C. Schoof and for very detailed and insightful comments made by the reviewer $\mathrm{C}$. Raymond.

Edited by: J. L. Bamber

\section{References}

Baral, D. R. and Hutter, C.: Asymptotic Theories of Ice Sheets and Ice Shelves, in: Geomorphological Fluid Mechanics, edited by Balmforth, N. J. and Provenzale, A., Lecture Notes in Physics, 11, 227-278, Springer, 2001.

Fowler, A. C.: Waves on glaciers, J. Fluid Mech., 120, 283-321, 1982.

Gudmundsson, G. H.: Transmission of basal variability to a glacier surface, Journal of Geophysical Research, 108(B5), 2253, doi:10.1029/2002JB002107, 2003.

Hindmarsh, R. C. A.: A numerical comparison of approximations to the Stokes equations used in ice sheet and glacier modeling, J. Geophys. Res., 109, F01012, doi:10.1029/2003JF000065, 2004.

Hutter, K.: Theoretical glaciology; material science of ice and the mechanics of glaciers and ice sheets, D. Reidel Publishing Company/Tokyo, Terra Scientific Publishing Company, 1983.

Jóhannesson, T.: Landscape of temperate ice caps, Ph.D. thesis, Univ. of Washington, 1992.

MacAyeal, D. R.: Large-scale ice flow over a viscous basal sediment: Theory and Application to Ice Stream B, Antarctica, J. Geophys. Res., 94, 4071-4078, 1989.

Morland, L. W.: Thermomechanical balances of ice-sheet flows, Geophysical and Astrophysical Fluid Dynamics, 29, 237-266, 1984.

Muszynski, I. and Birchfield, G. E.: A coupled marine ice-streamice - shelf model, J. Glaciology, 33, 3-14, 1987.

Nye, J. F.: The response of glaciers and ice-sheets to seasonal and climatic changes, Proceedings of the Royal Society of London, Ser. A, 256, 559-584, 1960.

Raymond, C. F.: Shear margins in glaciers and ice sheets, Journal of Glaciology, 42, 90-102, 1996.

Raymond, M. and Gudmundsson, G. H.: On the relationship between surface and basal properties on glaciers, ice sheets, and ice streams., Journal of Geophysical Research, 110, B08411, doi: 10.1029/2005JB003681, 2005.

Reeh, N.: Steady-state three-dimensional ice flow over an undulating base: first-order theory with linear ice rheology, J. Glaciology, 33, 177-185, 1987.

Schoof, C.: A note on inverting ice-stream surface data, J. Glaciology, 51, 181-182, 2005.

Schoof, C.: A variational approach to ice stream flow, Journal of Fluid Mechanics, 556, 227-251, doi:10.1017/ SS0022112006009591, 2006. 